\title{
SUFFICIENT SECOND-ORDER OPTIMALITY CONDITIONS FOR SEMILINEAR CONTROL PROBLEMS WITH POINTWISE STATE CONSTRAINTS*
}

\author{
EDUARDO CASAS ${ }^{\dagger}$, JUAN CARLOS DE LOS REYES ${ }^{\ddagger}$, AND FREDI TRÖLTZSCH ${ }^{\S}$
}

\begin{abstract}
Second-order sufficient optimality conditions are established for the optimal control of semilinear elliptic and parabolic equations with pointwise constraints on the control and the state. In contrast to former publications on this subject, the cone of critical directions is the smallest possible in the sense that the second-order sufficient conditions are the closest to the associated necessary ones. The theory is developed for elliptic distributed controls in domains up to dimension three. Moreover, problems of elliptic boundary control and parabolic distributed control are discussed in spatial domains of dimension two and one, respectively.
\end{abstract}

Key words. optimal control, elliptic equations, parabolic equations, pointwise state constraints, second-order necessary optimality conditions, second-order sufficient optimality conditions

AMS subject classifications. 49K20, 90C48

DOI. $10.1137 / 07068240 \mathrm{X}$

1. Introduction. In this paper, we essentially improve the theory of secondorder sufficient optimality conditions for state-constrained optimal control problems of elliptic and parabolic type. We derive second-order sufficient conditions that are as close as possible to the associated necessary ones. In this way, we are able to complete the theory of second-order sufficient conditions for this class of problems, if the dimension of the spatial domain is sufficiently small.

For the theory of nonconvex differentiable mathematical programming in finitedimensional spaces, second-order sufficient optimality conditions are indispensible both in the numerical analysis and for reliable numerical methods. If second-order information is not available, then local minima will not in general be stable and numerical methods will most likely not converge. For instance, the convergence analysis of SQP methods relies heavily on second-order conditions.

In the numerical analysis of nonlinear optimal control problems, second-order sufficient optimality conditions are even more important. If they are not satisfied, then the (strong) convergence of optimal controls or states and/or error estimates for numerical discretizations of the problems can hardly be shown. Also, other types of perturbations are difficult to handle without second-order conditions.

As is well known from the calculus of variations and the control theory for nonlinear ordinary differential equations, the theory of second-order conditions is more delicate and rich in function spaces. We mention, for instance, the work by Maurer [20] or Maurer and Zowe [21]. In particular, the well-known two-norm discrepancy occurs that essentially complicates the analysis; cf. the expositions in Ioffe [16] or

*Received by the editors February 8, 2007; accepted for publication (in revised form) January 4, 2008; published electronically July 2, 2008.

http://www.siam.org/journals/siopt/19-2/68240.html

${ }^{\dagger}$ Dpto. de Matemática Aplicada y Ciencias de la Computación, E.T.S.I. Industriales y de Telecomunicación, Universidad de Cantabria, E-39005 Santander, Spain (eduardo.casas@unican.es).This author was partially supported by Ministerio de Educación y Ciencia and "Ingenio Mathematica (i-MATH)" (Consolider Ingenio 2010 Spain).

‡Dpto. de Matemática, EPN Quito, Quito, Ecuador (jcdelosreyes@math.epn.edu.ec).

§Institut für Mathematik, Technische Universität Berlin, D-10623 Berlin, Germany (troeltzsch@ math.tu-berlin.de). 
Malanowski [18]. For the important but more difficult case of pointwise state constraints in the control of ordinary differential equations, we refer to Malanowski [19] and to the references therein.

At present, the control of distributed parameter systems with pointwise state constraints is a very active field of research. Although the majority of papers are still devoted to convex problems with linear equations, the important case of nonlinear state equations is attracting more interest. Here, second-order conditions are needed. However, when pointwise state constraints are imposed, the situation is more complicated, since the Lagrange multipliers associated with them are measures. In contrast to the theory for ordinary differential equations, this causes severe restrictions on the dimension of the spatial domains of the equations and reduces the regularity of the adjoint state.

To our best knowledge, there exist only two contributions to the theory of secondorder sufficient conditions for distributed problems with pointwise state constraints. The elliptic case was discussed in [12], while parabolic problems were investigated in [22]. The method of these papers was inspired by the splitting technique used in [11]. When applied to pointwise state constraints, the cones of critical directions established by this technique are too large so that the second-order sufficient conditions are based on slightly too strong assumptions. Moreover, the method was fairly complicated.

For other contributions to second-order optimality conditions for distributed parameter systems, we mention, for instance, the work by Bonnans [3] and the exposition in the monography by Bonnans and Shapiro [4] on elliptic problems with control constraints. We also refer to [9], where second-order necessary optimality conditions were first treated for elliptic problems, [10] for an abstract framework with applications to elliptic and parabolic problems, and [7], where elliptic problems with control constraints and state constraints of integral type were considered Moreover, we refer to the references therein.

In this paper, the sufficiency of second-order conditions is proven by a method that is close to the theory of nonlinear optimization in finite-dimensional spaces. We establish a cone of critical directions that is sharp; i.e., it is the one closest to the cone for establishing second-order necessary conditions.

We present a detailed proof for the case of distributed elliptic problems in domains of spatial dimension $n \leq 3$. Moreover, we briefly sketch the extension of this result to elliptic boundary control problems for $n \leq 2$ and to the parabolic distributed case for $n=1$.

2. Problem statement. Let $\Omega$ be an open and bounded domain in $\mathbb{R}^{n}, n \leq 3$, with a Lipschitz boundary $\Gamma$. In this domain we consider the following state equation:

$$
\left\{\begin{aligned}
A y+f(x, y)=u & \text { in } \Omega, \\
y=0 & \text { on } \Gamma,
\end{aligned}\right.
$$

where $f: \Omega \times \mathbb{R} \longrightarrow \mathbb{R}$ is a Carathéodory function and $A$ denotes a second-order elliptic operator of the form

$$
A y(x)=-\sum_{i, j=1}^{n} \partial_{x_{j}}\left(a_{i j}(x) \partial_{x_{i}} y(x)\right)
$$

the coefficients $a_{i j} \in L^{\infty}(\Omega)$ satisfy

$$
\lambda_{A}\|\xi\|^{2} \leq \sum_{i, j=1}^{n} a_{i j}(x) \xi_{i} \xi_{j} \quad \forall \xi \in \mathbb{R}^{n} \text { for a.e. } x \in \Omega
$$


for some $\lambda_{A}>0$. In (2.1), the function $u$ denotes the control, and $y_{u}$ is the solution associated to the control $u$. We will state later the conditions leading to the existence and uniqueness of a solution of $(2.1)$ in $C(\bar{\Omega}) \cap H^{1}(\Omega)$.

In this paper, we study the following optimal control problem:

$$
(\mathrm{P})\left\{\begin{array}{l}
\min J(u)=\int_{\Omega} L\left(x, y_{u}(x), u(x)\right) d x \\
\text { subject to }\left(y_{u}, u\right) \in\left(C(\bar{\Omega}) \cap H^{1}(\Omega)\right) \times L^{\infty}(\Omega), \\
\alpha(x) \leq u(x) \leq \beta(x) \text { for a.e. } x \in \Omega, \\
g\left(x, y_{u}(x)\right) \leq 0 \quad \forall x \in K,
\end{array}\right.
$$

where $\alpha(x)<\beta(x)$ for almost all $x \in \Omega, \alpha, \beta \in L^{\infty}(\Omega)$, and $K \subset \bar{\Omega}$ is a compact set. Let us state the assumptions on the functions $L, f$, and $g$.

(A1) $f$ is of class $C^{2}$ with respect to the second variable:

$$
f(\cdot, 0) \in L^{2}(\Omega), \quad \frac{\partial f}{\partial y}(x, y) \geq 0 \quad \text { for a.e. } x \in \Omega,
$$

and for all $M>0$ there exists a constant $C_{f, M}>0$ such that

$$
\begin{gathered}
\left|\frac{\partial f}{\partial y}(x, y)\right|+\left|\frac{\partial^{2} f}{\partial y^{2}}(x, y)\right| \leq C_{f, M} \text { for a.e. } x \in \Omega \text { and }|y| \leq M \\
\left|\frac{\partial^{2} f}{\partial y^{2}}\left(x, y_{2}\right)-\frac{\partial^{2} f}{\partial y^{2}}\left(x, y_{1}\right)\right| \leq C_{f, M}\left|y_{2}-y_{1}\right| \text { for }\left|y_{1}\right|,\left|y_{2}\right| \leq M \text { and for a.e. } x \in \Omega .
\end{gathered}
$$

(A2) $L: \Omega \times(\mathbb{R} \times \mathbb{R}) \longrightarrow \mathbb{R}$ is a Carathéodory function of class $C^{2}$ with respect to the second and third variables, $L(\cdot, 0,0) \in L^{1}(\Omega)$, and for all $M>0$ there is a constant $C_{L, M}>0$ and a function $\psi_{M} \in L^{2}(\Omega)$ such that

$$
\begin{gathered}
\left|\frac{\partial L}{\partial u}(x, y, u)\right|+\left|\frac{\partial L}{\partial y}(x, y, u)\right| \leq \psi_{M}(x), \quad\left\|D_{(y, u)}^{2} L(x, y, u)\right\| \leq C_{L, M}, \\
\left\|D_{(y, u)}^{2} L\left(x, y_{2}, u_{2}\right)-D_{(y, u)}^{2} L\left(x, y_{1}, u_{1}\right)\right\| \leq C_{L, M}\left(\left|y_{2}-y_{1}\right|+\left|u_{2}-u_{1}\right|\right)
\end{gathered}
$$

for a.e. $x \in \Omega$ and $|y|,\left|y_{i}\right|,|u|,\left|u_{i}\right| \leq M, i=1,2$, where $D_{(y, u)}^{2} L$ denotes the second derivative of $L$ with respect to $(y, u)$.

(A3) The function $g: K \times \mathbb{R} \longrightarrow \mathbb{R}$ is continuous, of class $C^{2}$ with respect to the second variable and $\partial_{y} g$, and $\partial_{y}^{2} g$ are also continuous functions in $K \times \mathbb{R}$. Moreover we will assume that $g(x, 0)<0$ is satisfied for every $x \in K \cap \Gamma$.

The following result on the existence of a solution holds true for (2.1) as well as for the problem $(\mathrm{P})$.

TheOREm 2.1. Suppose that (A1) holds. Then, for every $u \in L^{2}(\Omega)$, the state equation (2.1) has a unique solution $y_{u} \in C(\bar{\Omega}) \cap H_{0}^{1}(\Omega)$. Furthermore, if $u_{k} \rightarrow u$ weakly in $L^{2}(\Omega)$, then $y_{u_{k}} \rightarrow y_{u}$ strongly in $C(\bar{\Omega}) \cap H_{0}^{1}(\Omega)$.

The existence of a unique solution of $(2.1)$ in $H^{1}(\Omega) \cap L^{\infty}(\Omega)$ is classical. It is a consequence of the monotonicity of $f$ with respect to the second component. The continuity of $y_{u}$ is also a well-known result; see, for instance, [15]. The continuity property is a consequence of the compactness of the inclusion $L^{2}(\Omega) \subset W^{-1, p}(\Omega)$ for any $p<6$ and the fact that data $u \in W^{-1, p}(\Omega)$, with $6 / 5<p<6$, provide solutions in $C(\bar{\Omega}) \cap H_{0}^{1}(\Omega)$, the mapping $u \rightarrow y_{u}$ being continuous between these spaces. 
THEOREM 2.2. Let the function $L$ be convex with respect to the third component and the set of feasible controls be nonempty. Then, under assumptions (A1)-(A3), the control problem $(\mathrm{P})$ has at least one solution.

The proof of this theorem can be obtained by standard arguments.

Remark 2.3. We should remark that the differentiability of the functions $f, L$, and $g$ is not necessary to prove the previous theorems. In fact, the only properties we need are the continuity of $g$ and $f$ with respect to the second variable, the continuity of $L$ with respect to the second and third variables, the monotonicity of $f$ with respect to $y$, the convexity of $L$ with respect to $u$, and, for every $M>0$, the existence of two functions $\phi_{f, M} \in L^{2}(\Omega)$ and $\phi_{L, M} \in L^{1}(\Omega)$ such that

$$
|f(x, y)| \leq \phi_{f, M}(x) \text { and }|L(x, y, u)| \leq \phi_{L, M}(x) \text { for a.e. } x \in \Omega \text { and }|y|,|u| \leq M \text {. }
$$

These properties are an immediate consequence of the assumptions (A1)-(A3).

We finish this section by recalling some results about the differentiability of the nonlinear mappings involved in the control problem. For the detailed proofs, the reader is referred to Casas and Mateos [7].

THEOREM 2.4. If (A1) holds, then the mapping $G: L^{2}(\Omega) \longrightarrow C(\bar{\Omega}) \cap H_{0}^{1}(\Omega)$, defined by $G(u)=y_{u}$, is of class $C^{2}$. Moreover, for all $v, u \in L^{2}(\Omega), z_{v}=G^{\prime}(u) v$ is defined as the solution of

$$
\left\{\begin{array}{l}
A z_{v}+\frac{\partial f}{\partial y}\left(x, y_{u}\right) z_{v}=v \text { in } \Omega \\
z_{v}=0 \text { on } \Gamma
\end{array}\right.
$$

Finally, for every $v_{1}, v_{2} \in L^{2}(\Omega), z_{v_{1} v_{2}}=G^{\prime \prime}(u) v_{1} v_{2}$ is the solution of

$$
\left\{\begin{aligned}
A z_{v_{1} v_{2}}+\frac{\partial f}{\partial y}\left(x, y_{u}\right) z_{v_{1} v_{2}}+\frac{\partial^{2} f}{\partial y^{2}}\left(x, y_{u}\right) z_{v_{1}} z_{v_{2}} & =0 \text { in } \Omega, \\
z_{v_{1} v_{2}} & =0 \text { on } \Gamma
\end{aligned}\right.
$$

where $z_{v_{i}}=G^{\prime}(u) v_{i}, i=1,2$.

Remark 2.5. This theorem shows why we assume $n \leq 3$ : To prove Theorem 4.1 on second-order sufficient conditions, we need the operator $G$ to be differentiable from $L^{2}(\Omega)$ to $C(\bar{\Omega})$. This result holds true only for $n \leq 3$.

The proof can be obtained by the implicit function theorem; see, for instance, [7, Thm. 2.5] for the proof in the case of a Neumann problem, which can be translated straightforwardly to the Dirichlet case.

TheOrem 2.6. Suppose that (A1) and (A2) hold. Then $J: L^{\infty}(\Omega) \rightarrow \mathbb{R}$ is a functional of class $C^{2}$. Moreover, for every $u, v, v_{1}, v_{2} \in L^{\infty}(\Omega)$,

$$
J^{\prime}(u) v=\int_{\Omega}\left(\frac{\partial L}{\partial u}\left(x, y_{u}, u\right)+\varphi_{0 u}\right) v d x
$$

and

$$
\begin{aligned}
J^{\prime \prime}(u) v_{1} v_{2}= & \int_{\Omega}\left[\frac{\partial^{2} L}{\partial y^{2}}\left(x, y_{u}, u\right) z_{v_{1}} z_{v_{2}}+\frac{\partial^{2} L}{\partial y \partial u}\left(x, y_{u}, u\right)\left(z_{v_{1}} v_{2}+z_{v_{2}} v_{1}\right)\right. \\
& \left.+\frac{\partial^{2} L}{\partial u^{2}}\left(x, y_{u}, u\right) v_{1} v_{2}-\varphi_{0 u} \frac{\partial^{2} f}{\partial y^{2}}\left(x, y_{u}\right) z_{v_{1}} z_{v_{2}}\right] d x
\end{aligned}
$$

Copyright $\odot$ by SIAM. Unauthorized reproduction of this article is prohibited. 
where $y_{u}=G(u)$ and $\varphi_{0 u} \in W^{2, p}(\Omega)$ is the unique solution of the problem

$$
\left\{\begin{array}{l}
A^{*} \varphi+\frac{\partial f}{\partial y}\left(x, y_{u}\right) \varphi=\frac{\partial L}{\partial y}\left(x, y_{u}, u\right) \text { in } \Omega \\
\varphi=0 \text { on } \Gamma
\end{array}\right.
$$

$A^{*}$ being the adjoint operator of $A$ and $z_{v_{i}}=G^{\prime}(u) v_{i}, i=1,2$.

Let us remark that the linear and quadratic functionals $J^{\prime}(u)$ and $J^{\prime \prime}(u)$ can be extended from $L^{\infty}(\Omega)$ to $L^{2}(\Omega)$ by the formulas (2.4) and (2.5). To check this point it is enough to use the assumptions (A1) and (A2). This extension will be used in the rest of the paper.

The previous theorem and the next one follow easily from Theorem 2.4 and the chain rule.

TheOrem 2.7. Suppose that (A1) and (A3) hold. Then the mapping $F: L^{2}(\Omega) \rightarrow$ $C(K)$, defined by $F(u)=g\left(\cdot, y_{u}(\cdot)\right)$, is of class $C^{2}$. Moreover, for every $u, v, v_{1}, v_{2} \in$ $L^{2}(\Omega)$,

$$
F^{\prime}(u) v=\frac{\partial g}{\partial y}\left(\cdot, y_{u}(\cdot)\right) z_{v}(\cdot)
$$

and

$$
F^{\prime \prime}(u) v_{1} v_{2}=\frac{\partial^{2} g}{\partial y^{2}}\left(\cdot, y_{u}(\cdot)\right) z_{v_{1}}(\cdot) z_{v_{2}}(\cdot)+\frac{\partial g}{\partial y}\left(\cdot, y_{u}(\cdot)\right) z_{v_{1} v_{2}}(\cdot)
$$

where $z_{v_{i}}=G^{\prime}(u) v_{i}, i=1,2$, and $z_{v_{1} v_{2}}=G^{\prime \prime}(u) v_{1} v_{2}$.

Remark 2.8. A functional $L$ that is very frequently appearing in the applications is given by

$$
L(x, y, u)=L_{0}(x, y)+\frac{N}{2} u^{2} .
$$

In this case, the functional $J$ is twice differentiable not only in $L^{\infty}(\Omega)$ but also in $L^{2}(\Omega)$. Indeed, $J: L^{2}(\Omega) \longrightarrow \mathbb{R}$ is of class $C^{2}$, and the derivatives are given by the expressions

$$
J^{\prime}(u) v=\int_{\Omega}\left(N u(x)+\varphi_{0 u}\right) v d x
$$

and

$$
J^{\prime \prime}(u) v_{1} v_{2}=\int_{\Omega}\left[\frac{\partial^{2} L_{0}}{\partial y^{2}}\left(x, y_{u}\right) z_{v_{1}} z_{v_{2}}+N v_{1} v_{2}-\varphi_{0 u} \frac{\partial^{2} f}{\partial y^{2}}\left(x, y_{u}\right) z_{v_{1}} z_{v_{2}}\right] d x .
$$

Remark 2.9. The adjoint state $\varphi_{0 u}$ allows us to get a simple expression of $J^{\prime}(u)$, but it is not the complete adjoint state of the control problem because the adjoint state equation (2.6) does not include the Lagrange multiplier associated to the state constraint; see (3.2) below for the full definition.

3. First-order optimality conditions. We define the Hamiltonian associated with problem $(\mathrm{P}), H^{\lambda}: \Omega \times \mathbb{R}^{3} \longrightarrow \mathbb{R}$, by

$$
H^{\lambda}(x, y, u, \varphi)=\lambda \cdot L(x, y, u)+\varphi[u-f(x, y)] .
$$

Copyright $@$ by SIAM. Unauthorized reproduction of this article is prohibited. 
We denote by $M(K)$ the Banach space of all real and regular Borel measures in $K$, which is identified with the dual space of $C(K)$.

In the rest of the paper, a local minimum of $(\mathrm{P})$ is assumed to be a local solution in the sense of the topology of $L^{\infty}(\Omega)$. More precisely, we will say that $\bar{u}$ is a local minimum or a local solution of (P) in the sense of $L^{q}(\Omega), 1 \leq q \leq \infty$, if it is an admissible control of $(\mathrm{P})$ and there exists $\varepsilon_{\bar{u}}>0$ such that the minimum of $J$ in the admissible set of $(\mathrm{P})$ intersected with the ball $\bar{B}_{\varepsilon_{\bar{u}}}(\bar{u}) \subset L^{q}(\Omega)$ is achieved at $\bar{u}$.

The following result concerning Pontryagin's principle for problem $(\mathrm{P})$ is well known; look into [8] and [17] as well as in the references therein for the proof.

THEOREM 3.1. Let $\bar{u}$ be a local solution of $(\mathrm{P})$, and suppose that the assumptions (A1)-(A3) hold. Then there exist a real number $\bar{\lambda} \geq 0$, a measure $\bar{\mu} \in M(K)$, and a function $\bar{\varphi} \in W_{0}^{1, s}(\Omega)$, for all $1 \leq s<n /(n-1)$, such that

$$
\begin{gathered}
\bar{\lambda}+\|\bar{\mu}\|>0 \\
\left\{\begin{array}{l}
A^{*} \bar{\varphi}+\frac{\partial f}{\partial y}(x, \bar{y}(x)) \bar{\varphi}=\bar{\lambda} \frac{\partial L}{\partial y}(x, \bar{y}, \bar{u})+\frac{\partial g}{\partial y}(x, \bar{y}(x)) \bar{\mu} \text { in } \Omega, \\
\bar{\varphi}=0 \text { on } \Gamma
\end{array}\right. \\
\int_{K}(z(x)-g(x, \bar{y}(x))) d \bar{\mu}(x) \leq 0 \quad \forall z \in C(K) \text { such that } z(x) \leq 0 \forall x \in K, \\
H^{\bar{\lambda}}(x, \bar{y}(x), \bar{u}(x), \bar{\varphi}(x))=\min _{t \in\left[\alpha_{\varepsilon_{\bar{u}}}(x), \beta_{\varepsilon_{\bar{u}}}(x)\right]} H^{\bar{\lambda}}(x, \bar{y}(x), t, \bar{\varphi}(x)) \text { for a.e. } x \in \Omega,
\end{gathered}
$$

where

$$
\alpha_{\varepsilon_{\bar{u}}}(x)=\max \left\{\alpha(x), \bar{u}(x)-\varepsilon_{\bar{u}}\right\} \quad \text { and } \quad \beta_{\varepsilon_{\bar{u}}}(x)=\min \left\{\beta(x), \bar{u}(x)+\varepsilon_{\bar{u}}\right\},
$$

assuming that $\bar{u}$ is a minimum of $(\mathrm{P})$ in the ball $\bar{B}_{\varepsilon_{\bar{u}}}(\bar{u}) \subset L^{\infty}(\Omega)$. Moreover, if the following linearized Slater condition holds:

$$
\begin{aligned}
& \exists u_{0} \in L^{\infty}(\Omega), \text { with } \alpha(x) \leq u_{0}(x) \leq \beta(x) \text { for a.e. } x \in \Omega, \text { such that } \\
& g(x, \bar{y}(x))+\frac{\partial g}{\partial y}(x, \bar{y}(x)) z_{u_{0}-\bar{u}}(x)<0 \quad \forall x \in K,
\end{aligned}
$$

where $\bar{y}$ is the state associated to $\bar{u}$ and $z_{u_{0}-\bar{u}}=G^{\prime}(\bar{u})\left(u_{0}-\bar{u}\right)$, then the choice $\bar{\lambda}=1$ can be made.

From now on, we take $\bar{\lambda}=1$ and denote the Hamilton function by $H:=H^{1}$.

Remark 3.2. Together with the inequality $g(x, \bar{y}(x)) \leq 0$, relation (3.3) is equivalent to the well-known complementarity conditions

$$
g(x, \bar{y}(x)) \leq 0 \forall x \in K, \quad \bar{\mu} \geq 0 \text { in } M(K), \text { and } \int_{K} g(x, \bar{y}(x)) d \bar{\mu}(x)=0 .
$$

It is also well known that (3.3) implies that $\bar{\mu}$ is a positive measure concentrated on the set of points

$$
K_{0}=\{x \in K: g(x, \bar{y}(x))=0\}
$$

see, for instance, the references given before the statement of the previous theorem. From this property and assumption (A3), we deduce that $\bar{\mu}(K \cap \Gamma)=0$. 
Remark 3.3. By using elementary calculus, we obtain from (3.4) that

$$
\frac{\partial H}{\partial u}(x, \bar{y}(x), \bar{u}(x), \bar{\varphi}(x))(k-\bar{u}(x)) \geq 0 \quad \forall k \in[\alpha(x), \beta(x)]
$$

and

$$
\frac{\partial^{2} H}{\partial u^{2}}(x, \bar{y}(x), \bar{u}(x), \bar{\varphi}(x)) \geq 0 \text { if } \frac{\partial H}{\partial u}(x, \bar{y}(x), \bar{u}(x), \bar{\varphi}(x))=0
$$

for a.e. $x \in \Omega$. On the other hand, notice that

$$
\frac{\partial^{2} L}{\partial u^{2}}(x, y, u)=\frac{\partial^{2} H}{\partial u^{2}}(x, y, u, \varphi) .
$$

The inequality (3.6) implies that

$$
\begin{cases}\frac{\partial H}{\partial u}(x, \bar{y}(x), \bar{u}(x), \bar{\varphi}(x)) \geq 0 \quad & \text { if } \quad \bar{u}(x)=\alpha(x), \\ \frac{\partial H}{\partial u}(x, \bar{y}(x), \bar{u}(x), \bar{\varphi}(x)) \leq 0 & \text { if } \quad \bar{u}(x)=\beta(x), \\ \frac{\partial H}{\partial u}(x, \bar{y}(x), \bar{u}(x), \bar{\varphi}(x))=0 & \text { if } \quad \alpha(x)<\bar{u}(x)<\beta(x) .\end{cases}
$$

Reciprocally we also deduce from (3.6) that

$$
\left\{\begin{array}{l}
\bar{u}(x)=\alpha(x) \quad \text { if } \quad \frac{\partial H}{\partial u}(x, \bar{y}(x), \bar{u}(x), \bar{\varphi}(x))>0 \\
\bar{u}(x)=\beta(x) \quad \text { if } \quad \frac{\partial H}{\partial u}(x, \bar{y}(x), \bar{u}(x), \bar{\varphi}(x))<0 .
\end{array}\right.
$$

The properties given by (3.8) and (3.9) are satisfied almost everywhere in $\Omega$.

Remark 3.4. If we consider $\bar{u}$ in Theorem 3.1 to be a local minimum of $(\mathrm{P})$ in the sense of $L^{q}(\Omega), 1 \leq q<+\infty$, then (3.4) can be written in the form (see [8])

$$
H^{\bar{\lambda}}(x, \bar{y}(x), \bar{u}(x), \bar{\varphi}(x))=\min _{t \in[\alpha(x), \beta(x)]} H^{\bar{\lambda}}(x, \bar{y}(x), t, \bar{\varphi}(x)) \text { for a.e. } x \in \Omega .
$$

Let us formulate the Lagrangian version of the optimality conditions (3.2)-(3.4). The Lagrange function $\mathcal{L}: L^{\infty}(\Omega) \times M(K) \longrightarrow \mathbb{R}$ associated to problem $(\mathrm{P})$ is defined by

$\mathcal{L}(u, \mu)=J(u)+\int_{K} g\left(x, y_{u}(x)\right) d \mu(x)=\int_{\Omega} L\left(x, y_{u}(x), u(x)\right) d x+\int_{K} g\left(x, y_{u}(x)\right) d \mu(x)$.

By using (2.4) we find that

$$
\frac{\partial \mathcal{L}}{\partial u}(u, \mu) v=\int_{\Omega}\left(\frac{\partial L}{\partial u}\left(x, y_{u}(x), u(x)\right)+\varphi_{u}(x)\right) v(x) d x=\int_{\Omega} H_{u}(x) v(x) d x,
$$

where

$$
H_{u}(x)=\frac{\partial H}{\partial u}\left(x, y_{u}(x), u(x), \varphi(x)\right)
$$

Copyright $@$ by SIAM. Unauthorized reproduction of this article is prohibited. 
and $\varphi_{u} \in W_{0}^{1, s}(\Omega)$, for all $1 \leq s<n /(n-1)$, is the solution of the Dirichlet problem

$$
\left\{\begin{array}{l}
A^{*} \varphi+\frac{\partial f}{\partial y}\left(x, y_{u}\right) \varphi=\frac{\partial L}{\partial y}\left(x, y_{u}, u\right)+\frac{\partial g}{\partial y}\left(x, y_{u}(x)\right) \mu \text { in } \Omega \\
\varphi=0 \text { on } \Gamma
\end{array}\right.
$$

Notice that the subscript $u$ in $y_{u}$ and $H_{u}$ has a different meaning. While $y_{u}$ is used to indicate that $y$ is the state associated with $u, H_{u}$ denotes the partial derivative of $H$ with respect to $u$. This short notation for partial derivatives is frequently used in the following and will not cause confusion. Later, we also write $H_{u u}, H_{y u}$, or $H_{y u}$ for $\partial^{2} H / \partial u^{2}, \partial^{2} H / \partial y \partial u$, etc. $\bar{H}_{u}(x)$.

If we insert $(\bar{y}(x), \bar{u}(x), \bar{\varphi}(x))$ into expression (3.12), then we denote $H_{u}(x)$ by

Now the inequality (3.6) along with (3.11) leads to

$$
\frac{\partial \mathcal{L}}{\partial u}(\bar{u}, \bar{\mu})(u-\bar{u}) \geq 0 \text { if } \alpha(x) \leq u(x) \leq \beta(x) \text { for a.e. } x \in \Omega
$$

for any local solution $\bar{u}$, where $\bar{y}$ is the associated state and $\bar{\varphi}$ is the adjoint state given by (3.2), provided that (3.5) holds.

Before finishing this section we provide the expression of the second derivative of the Lagrangian with respect to the control, which will be used in the next section. From (2.8) we get

$$
\begin{gathered}
\frac{\partial^{2} \mathcal{L}}{\partial u^{2}}(u, \mu) v_{1} v_{2}=J^{\prime \prime}(u) v_{1} v_{2} \\
+\int_{K}\left[\frac{\partial^{2} g}{\partial y^{2}}\left(x, y_{u}(x)\right) z_{v_{1}}(x) z_{v_{2}}(x)+\frac{\partial g}{\partial y}\left(x, y_{u}(x)\right) z_{v_{1} v_{2}}(x)\right] d \mu(x) .
\end{gathered}
$$

By (2.5), this is equivalent to

$$
\begin{aligned}
\frac{\partial^{2} \mathcal{L}}{\partial u^{2}}(u, \mu) v_{1} v_{2}= & \int_{\Omega}\left[\frac{\partial^{2} L}{\partial y^{2}}\left(x, y_{u}, u\right) z_{v_{1}} z_{v_{2}}+\frac{\partial^{2} L}{\partial y \partial u}\left(x, y_{u}, u\right)\left(z_{v_{1}} v_{2}+z_{v_{2}} v_{1}\right)\right. \\
& \left.+\frac{\partial^{2} L}{\partial u^{2}}\left(x, y_{u}, u\right) v_{1} v_{2}-\varphi_{u} \frac{\partial^{2} f}{\partial y^{2}}\left(x, y_{u}\right) z_{v_{1}} z_{v_{2}}\right] d x \\
& +\int_{K} \frac{\partial^{2} g}{\partial y^{2}}\left(x, y_{u}(x)\right) z_{v_{1}}(x) z_{v_{2}}(x) d \mu(x)
\end{aligned}
$$

where $\varphi_{u}$ is the solution of (3.13).

4. Second-order optimality conditions. Let $\bar{u}$ be a feasible control of problem (P) and $\bar{y}$ be the associated state. We assume that there exist $\bar{\mu} \in M(K)$ and $\bar{\varphi} \in W_{0}^{1, s}(\Omega), 1 \leq s<n /(n-1)$, such that (3.2)-(3.4) are satisfied. As in the previous section, we use the notation

$$
\bar{H}_{u}(x):=\frac{\partial H}{\partial u}(x, \bar{y}(x), \bar{u}(x), \bar{\varphi}(x)) .
$$

The partial derivative of $H$ with respect to $y$ at $(x, \bar{y}(x), \bar{u}(x), \bar{\varphi}(x))$ is denoted analogously by $\bar{H}_{y}(x)$. 
Associated with $\bar{u}$, we define the cone of critical directions by

$$
\begin{aligned}
& C_{\bar{u}}=\left\{h \in L^{2}(\Omega): h \text { satisfies }(4.1),(4.2), \text { and }(4.3)\right\}, \\
& h(x)=\left\{\begin{array}{l}
\geq 0 \text { if } \bar{u}(x)=\alpha(x), \\
\leq 0 \text { if } \bar{u}(x)=\beta(x), \\
=0 \text { if } \bar{H}_{u}(x) \neq 0
\end{array}\right. \\
& \frac{\partial g}{\partial y}(x, \bar{y}(x)) z_{h}(x) \leq 0 \text { if } g(x, \bar{y}(x))=0, \\
& \int_{K} \frac{\partial g}{\partial y}(x, \bar{y}(x)) z_{h}(x) d \bar{\mu}(x)=0 .
\end{aligned}
$$

If we think in terms of the finite-dimensional case, inequality (4.2) says that the derivative of the state constraint in the direction $h$ is nonpositive if the constraint is active, and (4.3) states that this derivative is zero whenever the corresponding Lagrange multiplier is strictly positive. The relations (4.2)-(4.3) provide a convenient extension of the usual conditions in the finite-dimensional case.

We should mention that (4.3) is new in the context of infinite-dimensional optimization problems. In earlier papers on this subject, other extensions to the infinitedimensional case were suggested. For instance, Maurer and Zowe [21] used first-order sufficient conditions to consider strict positivity of Lagrange multipliers. Inspired by their approach, in [12] an application to state-constrained elliptic boundary control was suggested. In terms of our problem, (4.3) was relaxed by

$$
\int_{K} \frac{\partial g}{\partial y}(x, \bar{y}(x)) z_{h}(x) d \bar{\mu}(x) \geq-\varepsilon \int_{\Omega \backslash \Omega^{\tau}}|h(x)| d x
$$

for some $\varepsilon>0$; cf. [12, ineq. (5.15)]. Here $\Omega^{\tau} \subset \Omega$ is the set of points where $\left|\bar{H}_{u}(x)\right| \geq \tau$ holds true. We will prove that this relaxation is not necessary, which leads to a smaller cone of critical directions that seems to be optimal.

The sufficient second-order optimality conditions are given by the expressions (4.4) and (4.5) in the next theorem.

THEOREM 4.1. Let $\bar{u}$ a feasible control of problem $(\mathrm{P}), \bar{y}$ be the associated state, and $(\bar{\varphi}, \bar{\mu}) \in W_{0}^{1, s}(\Omega) \times M(K)$, for all $1 \leq s<n /(n-1)$, satisfying (3.2)-(3.4). Assume further that there exist two constants $\omega>0$ and $\tau>0$ such that

$$
\begin{aligned}
& \frac{\partial^{2} L}{\partial u^{2}}(x, \bar{y}(x), \bar{u}(x)) \geq \omega \text { if }\left|\bar{H}_{u}(x)\right| \leq \tau \text { for a.e. } x \in \Omega, \\
& \frac{\partial^{2} \mathcal{L}}{\partial u^{2}}(\bar{u}, \bar{\mu}) h^{2}>0 \quad \forall h \in C_{\bar{u}} \backslash\{0\} .
\end{aligned}
$$

Then there exist $\varepsilon>0$ and $\delta>0$ such that for every admissible control $u$ of problem (P) the following inequality holds:

$$
J(\bar{u})+\frac{\delta}{2}\|u-\bar{u}\|_{L^{2}(\Omega)}^{2} \leq J(u) \quad \text { if } \quad\|u-\bar{u}\|_{L^{\infty}(\Omega)}<\varepsilon .
$$

Remark 4.2. Thanks to (3.8), we can compare the second-order necessary condition (3.7) with the sufficient one given by (4.4). We do not require only the strict positivity on the second derivative of the Hamiltonian with respect to the control at the points where the first derivative vanishes, as in the finite-dimensional case. We 
also impose the second derivative to be strictly positive whenever the first derivative is "small." This is the usual case when we pass from finite to infinite dimension. For an instructive example the reader is referred to [14].

Inequality (4.4) is satisfied if the second derivative of $L$ with respect to $u$ is strictly positive for any $(y, u, \varphi) \in \mathbb{R}^{3}$ and almost all $x \in \Omega$. This assumption implies that $L$ is strictly convex with respect to $u$. We recall that the convexity of $L$ with respect to $u$ was necessary to prove the existence of an optimal control. Under this strict convexity assumption, the sufficient second-order optimality conditions are reduced to (4.5). This is the case when $L(x, y, u)=L_{0}(x, y)+N u^{2} / 2$ if $N>0$.

The condition (4.5) seems to be natural. In fact, under some regularity assumption, we can expect the inequality

$$
\frac{\partial^{2} \mathcal{L}}{\partial u^{2}}(\bar{u}, \bar{\mu}) h^{2} \geq 0 \quad \forall h \in C_{\bar{u}}
$$

to be a necessary condition for local optimality. At least this is the case when the state constraints are of integral type (see [7]) or when $K$ is a finite set of points (see $[6])$.

Proof of Theorem 4.1. We argue by contradiction. Suppose that $\bar{u}$ does not satisfy the quadratic growth condition (4.6). Then there exists a sequence $\left\{u_{k}\right\}_{k=1}^{\infty} \subset L^{2}(\Omega)$ of feasible controls of $(\mathrm{P})$ such that $u_{k} \rightarrow \bar{u}$ in $L^{\infty}(\Omega)$ and

$$
J(\bar{u})+\frac{1}{k}\left\|u_{k}-\bar{u}\right\|_{L^{2}(\Omega)}^{2}>J\left(u_{k}\right) \quad \forall k .
$$

Let us take

$$
\rho_{k}=\left\|u_{k}-\bar{u}\right\|_{L^{2}(\Omega)} \quad \text { and } \quad h_{k}=\frac{1}{\rho_{k}}\left(u_{k}-\bar{u}\right) .
$$

Since $\left\|h_{k}\right\|_{L^{2}(\Omega)}=1$, we can extract a subsequence, denoted in the same way, such that $h_{k} \rightarrow h$ weakly in $L^{2}(\Omega)$. Now we split the proof into several steps.

Step 1: $\frac{\partial \mathcal{L}}{\partial u}(\bar{u}, \bar{\mu}) h=0$. In the following, we write $y_{k}=y_{u_{k}}$. Since $u_{k}$ is feasible, it holds that $g\left(x, y_{k}(x)\right) \leq 0$ for every $x \in K$. By using (3.3) and (4.7) we obtain

$$
J(\bar{u})+\frac{1}{k}\left\|u_{k}-\bar{u}\right\|_{L^{2}(\Omega)}^{2}=\mathcal{L}(\bar{u}, \bar{\mu})+\frac{1}{k}\left\|u_{k}-\bar{u}\right\|_{L^{2}(\Omega)}^{2}>J\left(u_{k}\right) \geq \mathcal{L}\left(u_{k}, \bar{\mu}\right) .
$$

From the mean value theorem we know that

$$
\mathcal{L}\left(u_{k}, \bar{\mu}\right)=\mathcal{L}(\bar{u}, \bar{\mu})+\rho_{k} \frac{\partial \mathcal{L}}{\partial u}\left(v_{k}, \bar{\mu}\right) h_{k},
$$

with $v_{k}$ a point between $\bar{u}$ and $u_{k}$. This identity and (4.8) imply that

$$
\frac{\partial \mathcal{L}}{\partial u}\left(v_{k}, \bar{\mu}\right) h_{k}<\frac{1}{k \rho_{k}}\left\|u_{k}-\bar{u}\right\|_{L^{2}(\Omega)}^{2}=\frac{1}{k}\left\|u_{k}-\bar{u}\right\|_{L^{2}(\Omega)} .
$$

Since $h_{k} \rightarrow h$ weakly in $L^{2}(\Omega), v_{k} \rightarrow \bar{u}$ in $L^{\infty}(\Omega), y_{v_{k}} \rightarrow \bar{y}$ in $C(\bar{\Omega}) \cap H_{0}^{1}(\Omega)$, and $\varphi_{v_{k}} \rightarrow \bar{\varphi}$ in $W_{0}^{1, s}(\Omega) \subset L^{2}(\Omega)$ for $s$ close to $n /(n-1)$, we deduce from the above inequality and the expression of the derivative of the Lagrangian given by (3.11) that

$$
\frac{\partial \mathcal{L}}{\partial u}(\bar{u}, \bar{\mu}) h=\lim _{k \rightarrow \infty} \frac{\partial \mathcal{L}}{\partial u}\left(v_{k}, \bar{\mu}\right) h_{k} \leq 0
$$

Copyright (C) by SIAM. Unauthorized reproduction of this article is prohibited. 
On the other hand, since $\alpha(x) \leq u_{k}(x) \leq \beta(x)$ holds for almost all $x \in \Omega$, we deduce from the variational inequality (3.14) that

$$
\frac{\partial \mathcal{L}}{\partial u}(\bar{u}, \bar{\mu}) h_{k}=\rho_{k} \frac{\partial \mathcal{L}}{\partial u}(\bar{u}, \bar{\mu})\left(u_{k}-\bar{u}\right) \geq 0
$$

which implies that

$$
\frac{\partial \mathcal{L}}{\partial u}(\bar{u}, \bar{\mu}) h=\lim _{k \rightarrow \infty} \frac{\partial \mathcal{L}}{\partial u}\left(v_{k}, \bar{\mu}\right) h_{k} \geq 0
$$

This inequality, along with (4.9), leads to

$$
\frac{\partial \mathcal{L}}{\partial u}(\bar{u}, \bar{\mu}) h=0
$$

Step 2: $h \in C_{\bar{u}}$. We have to confirm (4.1)-(4.3). The set of functions of $L^{2}(\Omega)$ that are nonnegative if $\bar{u}(x)=\alpha(x)$ and nonpositive if $\bar{u}(x)=\beta(x)$, almost everywhere, is convex and closed. Therefore, it is weakly closed. Moreover $u_{k}-\bar{u}$ obviously belongs to this set, and thus every $h_{k}$ also does. Consequently, $h$ belongs to the same set. Then (3.10), together with (3.12), implies that

$$
\int_{\Omega}\left|\bar{H}_{u}(x) h(x)\right| d x=\int_{\Omega} \bar{H}_{u}(x) h(x) d x=\frac{\partial \mathcal{L}}{\partial u}(\bar{u}, \bar{\mu}) h=0,
$$

and hence $h(x)=0$ if $\bar{H}_{u}(x) \neq 0$, which concludes the proof of (4.1).

Let us prove (4.2). From Theorem 2.4 we have

$$
z_{h}=G^{\prime}(\bar{u}) h=\lim _{k \rightarrow \infty} \frac{\left(y_{\bar{u}+\rho_{k} h_{k}}-\bar{y}\right)}{\rho_{k}} \text { in } C(\bar{\Omega}) \cap H_{0}^{1}(\Omega),
$$

which implies for every $x \in K$ such that $g(x, \bar{y}(x))=0$ that

$$
\frac{\partial g}{\partial y}(x, \bar{y}(x)) z_{h}(x)=\lim _{k \rightarrow \infty} \frac{\left[g\left(x, y_{\bar{u}+\rho_{k} h_{k}}(x)\right)-g(x, \bar{y}(x))\right]}{\rho_{k}} \leq 0 .
$$

The last inequality follows from the fact that $u_{k}$ is feasible, $\bar{u}+\rho_{k} h_{k}=u_{k}$, and consequently $g\left(x, y_{\bar{u}+\rho_{k} h_{k}}(x)\right)=g\left(x, y_{u_{k}}(x)\right) \leq 0$ for every $x \in K$.

Finally, we prove (4.3). By taking $z=g\left(\cdot, y_{u_{k}}(\cdot)\right)$ in (3.3), we get

$$
\begin{gathered}
\int_{K} \frac{\partial g}{\partial y}(x, \bar{y}(x)) z_{h}(x) d \bar{\mu}(x)=\lim _{k \rightarrow \infty} \frac{1}{\rho_{k}} \int_{K}\left[g\left(x, y_{\bar{u}+\rho_{k} h_{k}}(x)\right)-g(x, \bar{y}(x))\right] d \bar{\mu}(x) \\
=\lim _{k \rightarrow \infty} \frac{1}{\rho_{k}} \int_{K}\left[g\left(x, y_{u_{k}}(x)\right)-g(x, \bar{y}(x))\right] d \bar{\mu}(x) \leq 0 .
\end{gathered}
$$

On the other hand, from (4.7) we find

$$
J^{\prime}(\bar{u}) h=\lim _{k \rightarrow \infty} \frac{J\left(\bar{u}+\rho_{k} h_{k}\right)-J(\bar{u})}{\rho_{k}}=\lim _{k \rightarrow \infty} \frac{J\left(u_{k}\right)-J(\bar{u})}{\rho_{k}} \leq \lim _{k \rightarrow \infty} \frac{\rho_{k}}{k}=0 .
$$

Then (4.10), (4.12), (4.13), and the fact that

$$
\frac{\partial \mathcal{L}}{\partial u}(\bar{u}, \bar{\mu}) h=J^{\prime}(\bar{u}) h+\int_{K} \frac{\partial g}{\partial y}(x, \bar{y}(x)) z_{h}(x) d \bar{\mu}(x)
$$

Copyright (c) by SIAM. Unauthorized reproduction of this article is prohibited. 
imply that

$$
J^{\prime}(\bar{u}) h=\int_{K} \frac{\partial g}{\partial y}(x, \bar{y}(x)) z_{h}(x) d \bar{\mu}(x)=0 .
$$

Thus (4.3) holds, and we know that $h \in C_{\bar{u}}$.

Step 3: $h=0$. By taking into account (4.5), it is enough to prove that

$$
\frac{\partial^{2} \mathcal{L}}{\partial u^{2}}(\bar{u}, \bar{\mu}) h \leq 0
$$

For this purpose, we evaluate the Lagrangian. By a second-order Taylor expansion, we derive

$$
\mathcal{L}\left(u_{k}, \bar{\mu}\right)=\mathcal{L}(\bar{u}, \bar{\mu})+\rho_{k} \frac{\partial \mathcal{L}}{\partial u}(\bar{u}, \bar{\mu}) h_{k}+\frac{\rho_{k}^{2}}{2} \frac{\partial^{2} \mathcal{L}}{\partial u^{2}}\left(w_{k}, \bar{\mu}\right) h_{k}^{2},
$$

$w_{k}$ being an intermediate point between $\bar{u}$ and $u_{k}$. From here we get

$$
\begin{array}{r}
\rho_{k} \frac{\partial \mathcal{L}}{\partial u}(\bar{u}, \bar{\mu}) h_{k}+\frac{\rho_{k}^{2}}{2} \frac{\partial^{2} \mathcal{L}}{\partial u^{2}}(\bar{u}, \bar{\mu}) h_{k}^{2} \\
=\mathcal{L}\left(u_{k}, \bar{\mu}\right)-\mathcal{L}(\bar{u}, \bar{\mu})+\frac{\rho_{k}^{2}}{2}\left[\frac{\partial^{2} \mathcal{L}}{\partial u^{2}}(\bar{u}, \bar{\mu})-\frac{\partial^{2} \mathcal{L}}{\partial u^{2}}\left(w_{k}, \bar{\mu}\right)\right] h_{k}^{2} .
\end{array}
$$

Now (4.8) can be written

$$
\mathcal{L}\left(u_{k}, \bar{\mu}\right)-\mathcal{L}(\bar{u}, \bar{\mu}) \leq \frac{\rho_{k}^{2}}{k}
$$

On the other hand, by taking into account the expression (3.15) of the second derivative of the Lagrangian, assumptions (A1)-(A3) and Theorems 2.1 and 2.4, and the fact that $u_{k} \rightarrow \bar{u}$ in $L^{\infty}(\Omega)$ and $\left\|h_{k}\right\|_{L^{2}(\Omega)}=1$, we obtain

$$
\begin{gathered}
\left|\left[\frac{\partial^{2} \mathcal{L}}{\partial u^{2}}(\bar{u}, \bar{\mu})-\frac{\partial^{2} \mathcal{L}}{\partial u^{2}}\left(w_{k}, \bar{\mu}\right)\right] h_{k}^{2}\right| \leq\left\|\frac{\partial^{2} \mathcal{L}}{\partial u^{2}}(\bar{u}, \bar{\mu})-\frac{\partial^{2} \mathcal{L}}{\partial u^{2}}\left(w_{k}, \bar{\mu}\right)\right\|_{B\left(L^{2}(\Omega)\right)}\left\|h_{k}\right\|_{L^{2}(\Omega)}^{2} \\
\quad=\left\|\frac{\partial^{2} \mathcal{L}}{\partial u^{2}}(\bar{u}, \bar{\mu})-\frac{\partial^{2} \mathcal{L}}{\partial u^{2}}\left(w_{k}, \bar{\mu}\right)\right\|_{B\left(L^{2}(\Omega)\right)} \rightarrow 0 \quad \text { when } k \rightarrow \infty
\end{gathered}
$$

where $B\left(L^{2}(\Omega)\right)$ is the space of quadratic forms in $L^{2}(\Omega)$.

Let us define

$$
\Omega^{\tau}=\left\{x \in \Omega:\left|\bar{H}_{u}(x)\right|>\tau\right\} .
$$

From (3.10) and the definition of $h_{k}$ we know that $\bar{H}_{u}(x) h_{k}(x) \geq 0$ in $\Omega$; therefore

$$
\frac{\partial \mathcal{L}}{\partial u}(\bar{u}, \bar{\mu}) h_{k}=\int_{\Omega} \bar{H}_{u}(x) h_{k}(x) d x \geq \int_{\Omega^{\tau}}\left|\bar{H}_{u}(x)\right|\left|h_{k}(x)\right| d x \geq \tau \int_{\Omega^{\tau}}\left|h_{k}(x)\right| d x .
$$

For any $\varepsilon>0$ we can take $k_{\varepsilon}$ such that

$$
\left\|\rho_{k} h_{k}\right\|_{L^{\infty}(\Omega)}=\left\|\bar{u}-u_{k}\right\|_{L^{\infty}(\Omega)}<\varepsilon \quad \forall k \geq k_{\varepsilon} \quad \text { for a.e. } x \in \Omega,
$$


and therefore

$$
\frac{\rho_{k}^{2} h_{k}^{2}(x)}{\varepsilon} \leq \rho_{k}\left|h_{k}(x)\right| \quad \forall k \geq k_{\varepsilon} \quad \text { for a.e. } x \in \Omega .
$$

From this inequality and (4.19) it follows that

$$
\rho_{k} \frac{\partial \mathcal{L}}{\partial u}(\bar{u}, \bar{\mu}) h_{k} \geq \rho_{k} \tau \int_{\Omega^{\tau}}\left|h_{k}(x)\right| d x \geq \frac{\rho_{k}^{2} \tau}{\varepsilon} \int_{\Omega^{\tau}} h_{k}^{2}(x) d x .
$$

By collecting (4.16)-(4.18) and (4.20) and dividing by $\rho_{k}^{2} / 2$, we obtain for any $k \geq k_{\varepsilon}$

$$
\frac{2 \tau}{\varepsilon} \int_{\Omega^{\tau}} h_{k}^{2}(x) d x+\frac{\partial^{2} \mathcal{L}}{\partial u^{2}}(\bar{u}, \bar{\mu}) h_{k}^{2} \leq \frac{2}{k}+\left\|\frac{\partial^{2} \mathcal{L}}{\partial u^{2}}(\bar{u}, \bar{\mu})-\frac{\partial^{2} \mathcal{L}}{\partial u^{2}}\left(w_{k}, \bar{\mu}\right)\right\|_{B\left(L^{2}(\Omega)\right)} .
$$

Next, we study the left-hand side of this inequality. First of all let us notice that from (3.15) we obtain for any $v \in L^{2}(\Omega)$

$$
\begin{aligned}
\frac{\partial^{2} \mathcal{L}}{\partial u^{2}}(\bar{u}, \bar{\mu}) v^{2}= & \int_{\Omega}\left[\bar{H}_{u u}(x) v^{2}(x)+2 \bar{H}_{u y}(x) z_{v}(x) v(x)+\bar{H}_{y y}(x) z_{v}^{2}(x)\right] d x \\
& +\int_{K} \frac{\partial^{2} g}{\partial y^{2}}(x, \bar{y}(x)) z_{v}^{2}(x) d \bar{\mu}(x),
\end{aligned}
$$

where

$$
\bar{H}_{u u}(x)=\frac{\partial^{2} H}{\partial u^{2}}(x, \bar{y}(x), \bar{u}(x), \bar{\varphi}(x))
$$

and $\bar{H}_{u y}$ and $\bar{H}_{y y}$ are defined analogously. We also recall that

$$
\bar{H}_{u u}(x)=\frac{\partial^{2} L}{\partial u^{2}}(x, \bar{y}(x), \bar{u}(x)) .
$$

Then we have

$$
\begin{aligned}
\frac{2 \tau}{\varepsilon} \int_{\Omega^{\tau}} & h_{k}^{2}(x) d x+\frac{\partial^{2} \mathcal{L}}{\partial u^{2}}(\bar{u}, \bar{\mu}) h_{k}^{2} \\
= & \int_{\Omega^{\tau}}\left(\frac{2 \tau}{\varepsilon}+\bar{H}_{u u}(x)\right) h_{k}^{2}(x) d x+\int_{\Omega \backslash \Omega^{\tau}} \bar{H}_{u u}(x) h_{k}^{2}(x) d x \\
& +\int_{\Omega}\left[2 \bar{H}_{u y}(x) z_{h_{k}}(x) h_{k}(x)+\bar{H}_{y y}(x) z_{h_{k}}^{2}(x)\right] d x \\
& +\int_{K} \frac{\partial^{2} g}{\partial y^{2}}(x, \bar{y}(x)) z_{h_{k}}^{2}(x) d \bar{\mu}(x) .
\end{aligned}
$$

From assumptions (A1)-(A3) we deduce the existence of $C>0$ such that $\left|\bar{H}_{u u}(x)\right| \leq C$ for a.e. $x \in \Omega$. Therefore we can take $\varepsilon>0$ small enough so that the following inequality holds:

$$
\frac{2 \tau}{\varepsilon}+\bar{H}_{u u}(x) \geq \frac{2 \tau}{\varepsilon}-C>0 \text { for a.e. } x \in \Omega^{\tau} .
$$

Thus

$$
\liminf _{k \rightarrow \infty} \int_{\Omega^{\tau}}\left(\frac{2 \tau}{\varepsilon}+\bar{H}_{u u}(x)\right) h_{k}^{2}(x) d x \geq \int_{\Omega^{\tau}}\left(\frac{2 \tau}{\varepsilon}+\bar{H}_{u u}(x)\right) h^{2}(x) d x .
$$

Copyright $@$ by SIAM. Unauthorized reproduction of this article is prohibited. 
Moreover from (4.4) we have $\bar{H}_{u u}(x) \geq \omega>0$ in $\Omega \backslash \Omega^{\tau}$, and therefore we also get

$$
\liminf _{k \rightarrow \infty} \int_{\Omega \backslash \Omega^{\tau}} \bar{H}_{u u}(x) h_{k}^{2}(x) d x \geq \int_{\Omega \backslash \Omega^{\tau}} \bar{H}_{u u}(x) h^{2}(x) d x .
$$

Finally, by taking into account that $z_{h_{k}} \rightarrow z_{h}$ strongly in $C(\bar{\Omega}) \cap H_{0}^{1}(\Omega)$, we deduce from (4.21)-(4.24) and (4.18) that

$$
\begin{gathered}
\int_{\Omega^{\tau}}\left(\frac{2 \tau}{\varepsilon}+\bar{H}_{u u}(x)\right) h^{2}(x) d x+\int_{\Omega \backslash \Omega^{\tau}} \bar{H}_{u u}(x) h^{2}(x) d x \\
(4.26)+\int_{\Omega}\left[2 \bar{H}_{u y}(x) h(x) z_{h}(x)+\bar{H}_{y y}(x) z_{h}^{2}(x)\right] d x+\int_{K} \frac{\partial^{2} g}{\partial y^{2}}(x, \bar{y}(x)) z_{h}^{2}(x) d \bar{\mu}(x) \leq 0 .
\end{gathered}
$$

This expression can be written as follows:

$$
\frac{2 \tau}{\varepsilon} \int_{\Omega^{\tau}} h^{2}(x) d x+\frac{\partial^{2} \mathcal{L}}{\partial u^{2}}(\bar{u}, \bar{\mu}) h^{2} \leq 0
$$

which along with (4.5) and the fact that $h \in C_{\bar{u}}$ implies that $h=0$.

Step 4: $h_{k} \rightarrow 0$ strongly in $L^{2}(\Omega)$. We have already proved that $h_{k} \rightarrow 0$ weakly in $L^{2}(\Omega)$; therefore $z_{h_{k}} \rightarrow 0$ strongly in $C(\bar{\Omega}) \cap H_{0}^{1}(\Omega)$. By using (4.21) and (4.23) and the fact that $\left\|h_{k}\right\|_{L^{2}(\Omega)}=1$, we conclude that

$$
\begin{gathered}
0<\min \left\{\omega, \frac{2 \tau}{\varepsilon}-C\right\}=\min \left\{\omega, \frac{2 \tau}{\varepsilon}-C\right\} \limsup _{k \rightarrow \infty} \int_{\Omega} h_{k}^{2}(x) d x \\
\leq \limsup _{k \rightarrow \infty}\left\{\int_{\Omega^{\tau}}\left(\frac{2 \tau}{\varepsilon}+\bar{H}_{u u}(x)\right) h_{k}^{2}(x) d x+\int_{\Omega \backslash \Omega^{\tau}} \bar{H}_{u u}(x) h_{k}^{2}(x) d x\right\} \\
\leq \limsup _{k \rightarrow \infty}\left\{\frac{2}{k}+\left\|\frac{\partial^{2} \mathcal{L}}{\partial u^{2}}(\bar{u}, \bar{\mu})-\frac{\partial^{2} \mathcal{L}}{\partial u^{2}}\left(w_{k}, \bar{\mu}\right)\right\|_{B\left(L^{2}(\Omega)\right)}\right. \\
\left.-\int_{K} \frac{\partial^{2} g}{\partial y^{2}}(x, \bar{y}(x)) z_{h_{k}}^{2}(x) d \bar{\mu}(x)-\int_{\Omega}\left[2 \bar{H}_{u y}(x) z_{h_{k}}(x) h_{k}(x)+\bar{H}_{y y}(x) z_{h_{k}}^{2}(x)\right] d x\right\}=0 .
\end{gathered}
$$

Thus we have the contradiction.

There is a very interesting particular case of $(\mathrm{P})$ where Theorem 4.1 has a stronger formulation.

Theorem 4.3. Assume that $L(x, y, u)=L_{0}(x, y)+N u^{2} / 2$, with $N>0$. If $\bar{u}$ is a feasible control of problem $(\mathrm{P}), \bar{y}$ is the associated state, $(\bar{\varphi}, \bar{\mu}) \in W_{0}^{1, s}(\Omega) \times M(K)$, for all $1 \leq s<n /(n-1)$, and $(\bar{y}, \bar{u}, \bar{\varphi}, \bar{\mu})$ satisfies (3.2)-(3.4) and (4.5), then there exist $\varepsilon>0$ and $\delta>0$ such that for every admissible control $u$ of problem $(\mathrm{P})$ the following inequality holds:

$$
J(\bar{u})+\frac{\delta}{2}\|u-\bar{u}\|_{L^{2}(\Omega)}^{2} \leq J(u) \quad \text { if } \quad\|u-\bar{u}\|_{L^{2}(\Omega)}<\varepsilon .
$$

We have already mentioned in Remark 4.2 that the first-order optimality conditions along with (4.5) are sufficient for optimality when $L(x, y, u)=L_{0}(x, y)+N u^{2} / 2$, with $N>0$. But the above theorem includes more very important information. Relation (4.27) says that $\bar{u}$ is a strict local minimum of $(\mathrm{P})$ in $L^{2}(\Omega)$. The fact that the control appears linearly in the state equation and quadratically in the cost functional allows us to get sufficient optimality conditions for a local minimum not only in $L^{\infty}(\Omega)$ but also in $L^{2}(\Omega)$. This fact is very important in the analysis of stability and convergence of numerical algorithms to solve $(\mathrm{P})$. The proof of Theorem 4.3 follows the same arguments and steps as those given in the proof of Theorem 4.1. The essential fact is that the functional $J$ is of class $C^{2}$ in $L^{2}(\Omega)$; see Remark 2.8.

Copyright $@$ by SIAM. Unauthorized reproduction of this article is prohibited. 
5. Bilateral state constraints. In this section we will consider the extension of the control problem to the case of bilateral state constraints. More precisely we formulate the control problem as follows:

$$
(\mathrm{P})\left\{\begin{array}{l}
\min J(u)=\int_{\Omega} L\left(x, y_{u}(x), u(x)\right) d x \\
\text { subject to }\left(y_{u}, u\right) \in\left(C(\bar{\Omega}) \cap H^{1}(\Omega)\right) \times L^{\infty}(\Omega), \\
\alpha(x) \leq u(x) \leq \beta(x) \quad \text { for a.e. } \quad x \in \Omega, \\
g_{a}(x) \leq g\left(x, y_{u}(x)\right) \leq g_{b}(x) \quad \forall x \in K,
\end{array}\right.
$$

where $g_{a}, g_{b}: K \mapsto \mathbb{R}$ are continuous functions and $g_{a}(x)<g_{b}(x)$ for every $x \in K$. We assume the same hypotheses as in the previous sections. All of the previous theorems remain valid with some obvious modifications that we are going to mention. The Slater assumption required in Theorem 3.5 is now formulated as follows:

$$
\begin{aligned}
& \exists u_{0} \in L^{\infty}(\Omega), \text { with } \alpha(x) \leq u_{0}(x) \leq \beta(x) \text { for a.e. } x \in \Omega, \text { such that } \\
& g_{a}(x)<g(x, \bar{y}(x))+\frac{\partial g}{\partial y}(x, \bar{y}(x)) z_{u_{0}-\bar{u}}(x)<g_{b}(x) \quad \forall x \in K
\end{aligned}
$$

Under this assumption, Theorem 3.1 remains valid except for (3.3), which is written now in the following way:

$$
\int_{K}(z(x))-g(x, \bar{y}(x)) d \bar{\mu}(x) \leq 0 \forall z \in C(K), \text { with } g_{a}(x) \leq z(x) \leq g_{b}(x) \forall x \in K .
$$

From (5.2) we deduce that $\bar{\mu}$ is concentrated at the set of points $K_{0}$ where the state constraint is active:

$$
K_{0}=K_{-} \cup K_{+}=\left\{x \in K: g(x, \bar{y}(x))=g_{a}(x)\right\} \cup\left\{x \in K: g(x, \bar{y}(x))=g_{b}(x)\right\} .
$$

Now the Lagrange multiplier $\bar{\mu}$ is not necessarily a positive measure. However, its Jordan decomposition into nonnegative measures $\bar{\mu}^{+}, \bar{\mu}^{-}$is as follows:

$$
\bar{\mu}=\bar{\mu}^{+}-\bar{\mu}^{-}, \quad \text { with } \operatorname{supp}\left(\bar{\mu}^{+}\right) \subset K_{+} \text {and } \operatorname{supp}\left(\bar{\mu}^{-}\right) \subset K_{-} .
$$

The cone of critical directions $C_{\bar{u}}$ is formed by the functions $h \in L^{2}(\Omega)$ satisfying (4.1) and

$$
\begin{aligned}
& \frac{\partial g}{\partial y}(x, \bar{y}(x)) z_{h}(x) \leq 0 \text { if } g(x, \bar{y}(x))=g_{b}(x), \\
& \frac{\partial g}{\partial y}(x, \bar{y}(x)) z_{h}(x) \geq 0 \text { if } g(x, \bar{y}(x))=g_{a}(x), \\
& \int_{K}\left|\frac{\partial g}{\partial y}(x, \bar{y}(x)) z_{h}(x)\right| d|\bar{\mu}|(x)=0,
\end{aligned}
$$

where $|\bar{\mu}|=\bar{\mu}^{+}+\bar{\mu}^{-}$. Then Theorem 4.1 is still true, and the only changes of the proof appear in Steps 1 and 2. In particular, (4.8) can be rewritten with the help 
of (3.3) in the following way:

$$
\begin{gathered}
\mathcal{L}(\bar{u}, \bar{\mu})+\frac{1}{k}\left\|u_{k}-\bar{u}\right\|_{L^{2}(\Omega)}^{2}-\int_{K} g(x, \bar{y}(x)) d \bar{\mu}(x)=J(\bar{u})+\frac{1}{k}\left\|u_{k}-\bar{u}\right\|_{L^{2}(\Omega)}^{2} \\
>J\left(u_{k}\right) \geq \mathcal{L}\left(u_{k}, \bar{\mu}\right)-\int_{K} g(x, \bar{y}(x)) d \bar{\mu}(x) \geq \mathcal{L}\left(u_{k}, \bar{\mu}\right)
\end{gathered}
$$

and the proof can continue as in Theorem 4.1.

On the other hand, relation (4.11) in Step 2 must be replaced by

$$
\frac{\partial g}{\partial y}(x, \bar{y}(x)) z_{h}(x)= \begin{cases}\leq 0 & \forall x \in K_{+} \\ \geq 0 & \forall x \in K_{-}\end{cases}
$$

Relations (4.12) and (4.13) remain valid. Finally, by using (4.10) and (5.6) we deduce the identity (5.5) as follows:

$$
\int_{K}\left|\frac{\partial g}{\partial y}(x, \bar{y}(x)) z_{h}(x)\right| d|\bar{\mu}|(x)=-\int_{K} \frac{\partial g}{\partial y}(x, \bar{y}(x)) z_{h}(x) d \bar{\mu}(x)=J^{\prime}(\bar{u}) h=0 .
$$

\section{Elliptic boundary control.}

6.1. Problem statement. The method of the preceding sections can be extended to other types of equations in a straightforward way. Here we discuss the case of boundary control, while the next section is devoted to a one-dimensional distributed parabolic control problem. Instead of (2.1), we consider now

$$
\left\{\begin{aligned}
A y+f(x, y)=0 & \text { in } \Omega \\
\partial_{\nu} y+\gamma y=u & \text { on } \Gamma
\end{aligned}\right.
$$

where $\partial_{\nu}$ denotes the conormal-derivative associated with $A$ and $\gamma \in L^{\infty}(\Gamma)$ is nonnegative with $\gamma \not \equiv 0$. In contrast to section 1 , we assume here that $n=2$. We need this stronger assumption, since now the control-to-state mapping $G$ must be twice continuously differentiable from $L^{2}(\Gamma)$ to $C(\bar{\Omega})$; cf. Remark 2.5. The differential operator $A$ is defined as in section 1 .

We consider the optimal boundary control problem

$$
(\mathrm{PB})\left\{\begin{array}{l}
\min J(u)=\int_{\Omega} L\left(x, y_{u}(x)\right) d x+\int_{\Gamma} \ell\left(x, y_{u}(x), u(x)\right) d s(x) \\
\text { subject to }\left(y_{u}, u\right) \in\left(C(\bar{\Omega}) \cap H^{1}(\Omega)\right) \times L^{\infty}(\Gamma), \\
\alpha(x) \leq u(x) \leq \beta(x) \text { for a.e. } x \in \Gamma \\
g\left(x, y_{u}(x)\right) \leq 0 \quad \forall x \in K .
\end{array}\right.
$$

Here $\alpha$ and $\beta$ are now functions from $L^{\infty}(\Gamma)$, with $\alpha(x) \leq \beta(x)$ for a.a. $x \in \Gamma, d s$ denotes the surface measure on $\Gamma, y_{u}$ is the solution of (6.1) associated with $u \in L^{2}(\Gamma)$, and $K \subset \bar{\Omega}$ is again a compact set.

The following assumptions are imposed on the data: We assume (A1)-(A3) on $f, L$, and $g$ (where, of course, the dependence of $L$ on $u$ in (A2) is redundant). Moreover, we require the following.

(A4) The function $\ell: \Gamma \times(\mathbb{R} \times \mathbb{R}) \longrightarrow \mathbb{R}$ satisfies assumption (A2) with $\ell$ substituted for $L$ and $\Gamma$ substituted for $\Omega$. 
Remark 6.1. We confine ourselves to a linear boundary condition. An extension to a nonlinear condition of the type $\partial_{\nu} y+b(x, y)=u$ is possible under associated assumptions on $b$. On the other hand, the assumption $\gamma \not \equiv 0$, that allows us to deduce the existence of a unique solution of (6.1), can be replaced by

$$
\frac{\partial f}{\partial y}(x, t)>0 \text { for all } x \in E \text { and } t \in \mathbb{R}
$$

where $E$ is a measurable subset of $\Omega$ with a strictly positive measure.

The proof of the next theorems is completely analogous to that of Theorems 2.2 and 2.4; see Alibert and Raymond [1].

TheOREM 6.2. Suppose that (A1) holds. Then, for every $u \in L^{2}(\Gamma)$, the state equation (6.1) has a unique solution $y_{u} \in C(\bar{\Omega}) \cap H^{1}(\Omega)$. Furthermore, if $u_{k} \rightarrow u$ weakly in $L^{2}(\Gamma)$, then $y_{u_{k}} \rightarrow y_{u}$ strongly in $C(\bar{\Omega}) \cap H^{1}(\Omega)$.

Notice that controls of $L^{2}(\Gamma)$ are transformed continuously to states in the Hölder space $C^{0, \kappa}(\Omega)$, with some $0<\kappa<1$; cf. Stampacchia [23, Thm. 14.2]. The second part of the statement is an immediate conclusion.

THEOREM 6.3. Assume that (A1)-(A4) are fulfilled, the function $\ell$ is convex with respect to the third component, and the set of feasible controls is nonempty. Then the control problem $(\mathrm{PB})$ has at least one solution.

The proof can be performed by standard methods.

6.2. Necessary optimality conditions. We first state results on the firstand second-order derivatives of the control-to-state mapping $G(u)=y_{u}$ and of the reduced objective functional $J$. The results are analogous to Theorems 2.6-2.7 so that we only collect them without proof, since the associated modifications are obvious. Under assumptions (A1)-(A4), all mappings listed below are of class $C^{2}$ from $L^{\infty}(\Gamma)$ to their respective image spaces. The associated derivatives can be obtained as follows.

We define, for $v \in L^{2}(\Gamma)$, the function $z_{v}$ as the unique solution to

$$
\left\{\begin{array}{l}
A z_{v}+\frac{\partial f}{\partial y}\left(x, y_{u}\right) z_{v}=0 \text { in } \Omega \\
\partial_{\nu} z_{v}+\gamma z_{v}=v \text { on } \Gamma .
\end{array}\right.
$$

Then $G^{\prime}$ is given by $G^{\prime}(u) v=z_{v}$. Moreover, for $v_{1}, v_{2} \in L^{2}(\Gamma)$, we introduce $z_{v_{i}}=$ $G^{\prime}(u) v_{i}, i=1,2$, and obtain $G^{\prime \prime}(u) v_{1} v_{2}=z_{v_{1} v_{2}}$, where $z_{v_{1} v_{2}}$ is the solution to

$$
\left\{\begin{aligned}
A z_{v_{1} v_{2}}+\frac{\partial f}{\partial y}\left(x, y_{u}\right) z_{v_{1} v_{2}}+\frac{\partial^{2} f}{\partial y^{2}}\left(x, y_{u}\right) z_{v_{1}} z_{v_{2}} & =0 \text { in } \Omega \\
\partial_{\nu} z_{v_{1} v_{2}}+\gamma z_{v_{1} v_{2}} & =0 \text { on } \Gamma .
\end{aligned}\right.
$$

The adjoint state $\varphi_{0 u} \in H_{0}^{1}(\Omega)$ associated with $u$ and $J$ is introduced as the unique solution to

$$
\left\{\begin{array}{l}
A^{*} \varphi+\frac{\partial f}{\partial y}\left(x, y_{u}\right) \varphi=\frac{\partial L}{\partial y}\left(x, y_{u}\right) \text { in } \Omega \\
\partial_{\nu} \varphi+\gamma \varphi=\frac{\partial \ell}{\partial y}\left(x, y_{u}, u\right) \text { on } \Gamma .
\end{array}\right.
$$

Copyright $@$ by SIAM. Unauthorized reproduction of this article is prohibited. 
It holds that

$$
\begin{aligned}
& J^{\prime}(u) v=\int_{\Gamma}\left(\frac{\partial \ell}{\partial u}\left(x, y_{u}, u\right)+\varphi_{0 u}\right) v d s \\
J^{\prime \prime}(u) v_{1} v_{2}= & \int_{\Omega}\left[\frac{\partial^{2} L}{\partial y^{2}}\left(x, y_{u}, u\right) z_{v_{1}} z_{v_{2}}-\varphi_{0 u} \frac{\partial^{2} f}{\partial y^{2}}\left(x, y_{u}\right) z_{v_{1}} z_{v_{2}}\right] d x \\
& +\int_{\Gamma}\left[\frac{\partial^{2} \ell}{\partial y^{2}}\left(x, y_{u}, u\right) z_{v_{1}} z_{v_{2}}+\frac{\partial^{2} \ell}{\partial y \partial u}\left(x, y_{u}, u\right)\left(z_{v_{1}} v_{2}+z_{v_{2}} v_{1}\right)\right. \\
& \left.+\frac{\partial^{2} \ell}{\partial u^{2}}\left(x, y_{u}, u\right) v_{1} v_{2}\right] d s .
\end{aligned}
$$

Under (A1) and (A3), the mapping $F: L^{2}(\Gamma) \rightarrow C(K)$, defined by $F(u)=$ $g\left(\cdot, y_{u}(\cdot)\right)$, is of class $C^{2}$. For every $u, v, v_{1}, v_{2} \in L^{2}(\Gamma)$, its first- and second-order derivatives are given again by (2.7) and (2.8), respectively.

Now we introduce the Hamiltonian $H$ by

$$
H(x, y, u, \varphi)=\ell(x, y, u)+\varphi[u-\gamma y] .
$$

The first-order necessary conditions admit the following form.

THEOREM 6.4. Let $\bar{u}$ be a local solution of (PB). Suppose that assumptions (A1)(A4) hold, and assume the linearized Slater condition (3.5) with some $u_{0} \in L^{\infty}(\Gamma)$, $\alpha(x) \leq u_{0}(x) \leq \beta(x)$ for a.e. $x \in \Gamma$. Then there exist a measure $\bar{\mu} \in M(K)$ and a function $\bar{\varphi} \in W^{1, s}(\Omega)$ for all $1 \leq s<n /(n-1)$ such that

$$
\begin{gathered}
\left\{\begin{array}{l}
A^{*} \bar{\varphi}+\frac{\partial f}{\partial y}(x, \bar{y}(x)) \bar{\varphi}=\frac{\partial L}{\partial y}(x, \bar{y}, \bar{u})+\frac{\partial g}{\partial y}(x, \bar{y}(x)) \bar{\mu}_{\left.\right|_{\Omega}} \text { in } \Omega, \\
\partial_{\nu} \bar{\varphi}+\gamma \bar{\varphi}=\frac{\partial g}{\partial y}(x, \bar{y}(x)) \bar{\mu}_{\left.\right|_{\Gamma}} \text { on } \Gamma,
\end{array}\right. \\
\int_{K}(z(x)-g(x, \bar{y}(x))) d \bar{\mu}(x) \leq 0 \quad \forall z \in C(K) \text { such that } z(x) \leq 0 \forall x \in K, \\
H(x, \bar{y}(x), \bar{u}(x), \bar{\varphi}(x))=\min _{t \in\left[\alpha_{\varepsilon_{\bar{u}}}(x), \beta_{\varepsilon_{\bar{u}}}(x)\right]} H(x, \bar{y}(x), t, \bar{\varphi}(x)) \text { for a.e. } x \in \Gamma,
\end{gathered}
$$

where $\alpha_{\varepsilon_{\bar{u}}}$ and $\beta_{\varepsilon_{\bar{u}}}$ are defined similarly as in Theorem 3.1 and $\bar{\mu}_{\left.\right|_{\Omega}}$ and $\bar{\mu}_{\left.\right|_{\Gamma}}$ denote the restrictions of $\mu$ to $\Omega$ and $\Gamma$, respectively.

At the optimal point, the derivatives of $H$ fulfill the relations (3.6)-(3.10) with an obvious modification: We have to substitute $x \in \Gamma$ for $x \in \Omega$. Moreover, we have to replace (3.8) by

$$
\frac{\partial^{2} \ell}{\partial u^{2}}(x, y, u)=\frac{\partial^{2} H}{\partial u^{2}}(x, y, u, \varphi) .
$$

The Lagrangian function $\mathcal{L}: L^{\infty}(\Gamma) \times M(K) \longrightarrow \mathbb{R}$ associated to problem (PB) is defined by

$$
\mathcal{L}(u, \mu)=\int_{\Omega} L\left(x, y_{u}(x)\right) d x+\int_{\Gamma} \ell\left(x, y_{u}(x), u(x)\right) d s+\int_{K} g\left(x, y_{u}(x)\right) d \mu(x) .
$$

Copyright (c) by SIAM. Unauthorized reproduction of this article is prohibited. 
By using (6.6) we deduce that

$$
\frac{\partial \mathcal{L}}{\partial u}(u, \mu) v=\int_{\Gamma} H_{u}(x) v(x) d s
$$

where

$$
H_{u}(x)=\frac{\partial H}{\partial u}\left(x, y(x), u(x), \varphi_{u}(x)\right)
$$

and $\varphi_{u}$ is obtained from the adjoint equation (6.8), where $y_{u}$ is substituted for $\bar{y}, u$ for $\bar{u}$, and $\mu$ for $\bar{\mu}$, respectively. We finally indicate the expression for the second-order derivative of $\mathcal{L}$ :

$$
\begin{aligned}
\frac{\partial^{2} \mathcal{L}}{\partial u^{2}}(u, \mu) v_{1} v_{2}= & \int_{\Omega}\left[\frac{\partial^{2} L}{\partial y^{2}}\left(x, y_{u}\right) z_{v_{1}} z_{v_{2}}-\varphi_{u} \frac{\partial^{2} f}{\partial y^{2}}\left(x, y_{u}\right) z_{v_{1}} z_{v_{2}}\right] d x \\
& +\int_{\Gamma}\left[\frac{\partial^{2} \ell}{\partial y^{2}}\left(x, y_{u}, u\right) z_{v_{1}} z_{v_{2}}+\frac{\partial^{2} \ell}{\partial y \partial u}\left(x, y_{u}, u\right)\left(z_{v_{1}} v_{2}+z_{v_{2}} v_{1}\right)\right. \\
& \left.+\frac{\partial^{2} \ell}{\partial u^{2}}\left(x, y_{u}, u\right) v_{1} v_{2}\right] d s \\
& +\int_{K} \frac{\partial^{2} g}{\partial y^{2}}\left(x, y_{u}(x)\right) z_{v_{1}}(x) z_{v_{2}}(x) d \mu(x),
\end{aligned}
$$

where $\varphi_{u}$ is defined as after (6.13).

6.3. Second-order sufficient optimality conditions. Let $\bar{u}$ be a feasible control of problem (PB) and $\bar{y}$ be the associated state. We assume that there exist $\bar{\mu} \in M(K)$ and $\bar{\varphi} \in W_{0}^{1, s}(\Omega), 1 \leq s<n /(n-1)$, such that the first-order necessary conditions (6.8)-(6.10) are satisfied. Associated with $\bar{u}$, we introduce the function

$$
\bar{H}_{u}(x)=\frac{\partial H}{\partial u}(x, \bar{y}(x), \bar{u}(x), \bar{\varphi}(x))
$$

and define the cone of critical directions by

$$
C_{\bar{u}}=\left\{h \in L^{2}(\Gamma): h \text { satisfies (4.1)-(4.3) with } x \in \Gamma\right\} .
$$

Notice that this cone is only formally the same as in (4.1)-(4.3), since $x$ varies here through $\Gamma$. The second-order sufficient condition admits now the following form.

THEOREM 6.5. Assume that $n=2$, and let $\bar{u}$ be a feasible control of problem (PB), $\bar{y}$ the associated state, and $(\bar{\varphi}, \bar{\mu}) \in W^{1, s}(\Omega) \times M(K)$, for all $1 \leq s<n /(n-1)$, satisfying (6.8)-(6.10). Let there exist two constants $\omega>0$ and $\tau>0$ such that

$$
\begin{aligned}
& \frac{\partial^{2} \ell}{\partial u^{2}}(x, \bar{y}(x), \bar{u}(x)) \geq \omega \text { if }\left|\bar{H}_{u}(x)\right| \leq \tau \text { for a.e. } x \in \Gamma, \\
& \frac{\partial^{2} \mathcal{L}}{\partial u^{2}}(\bar{u}, \bar{\mu}) h^{2}>0 \quad \forall h \in C_{\bar{u}} \backslash\{0\},
\end{aligned}
$$

where $C_{\bar{u}}$ is defined in (6.15) and $\partial^{2} \mathcal{L} / \partial u^{2}$ is taken from (6.14), with $u:=\bar{u}$ and $\mu:=\bar{\mu}$.

Then there exist $\varepsilon>0$ and $\delta>0$ such that, for every admissible control $u$ of problem $(\mathrm{PB})$, the following inequality holds:

$$
J(\bar{u})+\frac{\delta}{2}\|u-\bar{u}\|_{L^{2}(\Gamma)}^{2} \leq J(u) \quad \text { if } \quad\|u-\bar{u}\|_{L^{\infty}(\Gamma)}<\varepsilon .
$$

Copyright $@$ by SIAM. Unauthorized reproduction of this article is prohibited. 
Proof. The proof is almost identical with the one of Theorem 4.1. Therefore, we mention only where essential changes occur.

Throughout the proof, we have to perform the obvious modification that $L^{2}(\Gamma)$, $L^{\infty}(\Gamma)$, and $H^{1}(\Omega)$ must be substituted for $L^{2}(\Omega), L^{\infty}(\Omega)$, and $H_{0}^{1}(\Omega)$, respectively. Moreover, in some integrals, $\Omega$ must obviously be replaced by $\Gamma$. Then Steps 1 and 2 can be adopted without further changes.

Step 3: The arguments up to (4.18) do not need changes. Next, we modify $\Omega^{\tau}$ by

$$
\Gamma^{\tau}=\left\{x \in \Gamma:\left|\bar{H}_{u}(x)\right|>\tau\right\}
$$

Hereafter, $\Omega$ and $\Omega^{\tau}$ are replaced by $\Gamma$ and $\Gamma^{\tau}$, respectively. In (4.22), $\ell$ must be substituted for $L$, and in (4.23) we add the integral over $\partial^{2} L / \partial u^{2}$ to arrive at

$$
\begin{aligned}
\frac{2 \tau}{\varepsilon} \int_{\Gamma^{\tau}} & h_{k}^{2}(x) d s+\frac{\partial^{2} \mathcal{L}}{\partial u^{2}}(\bar{u}, \bar{\mu}) h_{k}^{2} \\
= & \int_{\Gamma^{\tau}}\left(\frac{2 \tau}{\varepsilon}+\bar{H}_{u u}(x)\right) h_{k}^{2}(x) d s+\int_{\Gamma \backslash \Gamma^{\tau}} \bar{H}_{u u}(x) h_{k}^{2}(x) d s \\
& +\int_{\Gamma}\left[2 \bar{H}_{u y}(x) z_{h_{k}}(x) h_{k}(x)+\bar{H}_{y y}(x) z_{h_{k}}^{2}(x)\right] d s \\
& +\int_{K} \frac{\partial^{2} g}{\partial y^{2}}(x, \bar{y}(x)) z_{h_{k}}^{2}(x) d \bar{\mu}(x)+\int_{\Omega} \frac{\partial^{2} L}{\partial y^{2}}(x, \bar{y}(x)) z_{h_{k}}^{2}(x) d x .
\end{aligned}
$$

Analogously, this term must be added to the left-hand side of (4.26).

Step 4: First, we conclude from $h_{k} \rightarrow 0$ in $L^{2}(\Gamma)$ that $z_{h_{k}} \rightarrow 0$ strongly in $C(\bar{\Omega})$. Proceeding as in the former Step 4 , we finally conclude with

$$
\begin{aligned}
0< & \limsup _{k \rightarrow \infty}\left\{\frac{2}{k}+\left\|\frac{\partial^{2} \mathcal{L}}{\partial u^{2}}(\bar{u}, \bar{\mu})-\frac{\partial^{2} \mathcal{L}}{\partial u^{2}}\left(w_{k}, \bar{\mu}\right)\right\|_{B\left(L^{2}(\Gamma)\right)}\right. \\
& -\int_{K} \frac{\partial^{2} g}{\partial y^{2}}(x, \bar{y}(x)) z_{h_{k}}^{2}(x) d \bar{\mu}(x)-\int_{\Omega} \frac{\partial^{2} L}{\partial y^{2}}(x, \bar{y}(x)) z_{h_{k}}^{2}(x) d x \\
& \left.-\int_{\Omega}\left[2 \bar{H}_{u y}(x) z_{h_{k}}(x) h_{k}(x)+\bar{H}_{y y}(x) z_{h_{k}}^{2}(x)\right] d x\right\}=0 .
\end{aligned}
$$

\section{The parabolic case.}

7.1. Problem statement. Finally we prove that our method can also be extended to one-dimensional parabolic problems with distributed control. This extension is addressed here. To define the parabolic problem, we consider the onedimensional domain $\Omega=(a, b)$ and the time interval $[0, T]$ for given $T>0$. We fix an initial value $y_{0} \in C[a, b]$ and introduce the set $Q=(a, b) \times(0, T)$. Moreover, we introduce the space $W(0, T)=\left\{y \in L^{2}\left(0, T ; H^{1}(\Omega)\right): \frac{d y}{d t} \in L^{2}\left(0, T ; H^{1}(\Omega)^{\prime}\right)\right\}$.

Remark 7.1. Again, the restriction on the dimension of $\Omega$ comes from the requirement that the control-to-state mapping is of class $C^{2}$ from $L^{2}(Q)$ to $C(\bar{Q})$. This holds true only for $n=1$. We should mention here that boundary controls cannot be handled by our approach. Neumann boundary data from $L^{2}(0, T)$ are not, in general, transformed into continuous states. 
The parabolic equation is defined by

$$
\left\{\begin{aligned}
\frac{d y}{d t}+A y+f(x, t, y) & =u & & \text { in }(a, b) \times(0, T), \\
-\partial_{x} y(a, t) & =0 & & \text { in }(0, T), \\
\partial_{x} y(b, t) & =0 & & \text { in }(0, T), \\
y(\cdot, 0) & =y_{0} & & \text { in }(a, b),
\end{aligned}\right.
$$

where $\partial_{x}$ denotes the partial derivative with respect to $x$. The associated optimal control problem is

$$
(\mathrm{PP})\left\{\begin{aligned}
& \min J(u)= \int_{0}^{T} \int_{a}^{b} L\left(x, t, y_{u}(x, t), u(x, t)\right) d x d t+\int_{a}^{b} r(x, y(x, T)) d x \\
& \quad+\int_{0}^{T} \ell_{a}\left(t, y_{u}(a, t)\right) d t+\int_{0}^{T} \ell_{b}\left(t, y_{u}(b, t)\right) d t \\
& \text { subject to }\left(y_{u}, u\right) \in(C(\bar{Q}) \cap W(0, T)) \times L^{\infty}(Q), \\
& \alpha(x, t) \leq u(x, t) \leq \beta(x, t) \quad \text { for a.e. } \quad(x, t) \in Q, \\
& g\left(x, t, y_{u}(x, t)\right) \leq 0 \quad \forall(x, t) \in K .
\end{aligned}\right.
$$

Here $\alpha$ and $\beta$ are functions from $L^{\infty}(Q)$, with $\alpha(x, t) \leq \beta(x, t)$ for a.a. $(x, t) \in Q, y_{u}$ is the solution of (7.1) associated with $u \in L^{2}(Q)$, and $K \subset \bar{Q}$ is a compact set.

The following assumptions are required.

(A5) The function $f: Q \times \mathbb{R} \longrightarrow \mathbb{R}$ satisfies the modification of assumption (A1) that is obtained by substituting $Q$ for $\Omega$ and $(x, t)$ for $x$, respectively.

(A6) The function $L: Q \times(\mathbb{R} \times \mathbb{R}) \longrightarrow \mathbb{R}$ satisfies the modified assumption (A2) obtained by substituting $Q$ for $\Omega$ and $(x, t)$ for $x$, respectively.

(A7) The function $g: K \times \mathbb{R} \longrightarrow \mathbb{R}$ is continuous and is of class $C^{2}$ with respect to the second variable, and $\partial_{y} g$ and $\partial_{y}^{2} g$ are also continuous functions in $K \times \mathbb{R}$. Moreover, the strict inequality

$$
g\left(x, 0, y_{0}(x)\right)<0
$$

holds for every $x \in K \cap \bar{\Omega}$.

(A8) The functions $\ell_{k}:[0, T] \times \mathbb{R} \longrightarrow \mathbb{R}, k \in\{a, b\}$, are Carathéodory functions of class $C^{2}$ with respect to the second variable with $\ell_{k}(\cdot, 0) \in L^{1}(0, T)$. For all $M>0$, there exist a constant $C_{M}>0$ and a function $\psi_{M} \in L^{2}(0, T)$ such that

$$
\begin{gathered}
\left|\frac{\partial \ell_{k}}{\partial u}(t, y)\right| \leq \psi_{M}(x), \quad\left|\frac{\partial^{2} \ell_{k}}{\partial y^{2}}(t, y)\right| \leq C_{M}, \\
\left|\frac{\partial^{2} \ell_{k}}{\partial y^{2}}\left(t, y_{2}\right)-\frac{\partial^{2} \ell_{k}}{\partial y^{2}}\left(t, y_{1}\right)\right| \leq C_{M}\left|y_{2}-y_{1}\right|
\end{gathered}
$$

holds for $k \in\{a, b\}$ for a.e. $t \in[0, T]$ and $|y|,\left|y_{i}\right| \leq M, i=1,2$.

Analogously, $r:[a, b] \times \mathbb{R} \longrightarrow \mathbb{R}$ is a Carathéodory function of class $C^{2}$ with respect to the second variable with $r(\cdot, 0) \in L^{1}(a, b)$. It satisfies the assumptions on $\ell_{k}$ above with $\ell_{k}$ replaced by $r,(a, b)$ substituted for $(0, T)$, and $x$ substituted for $t$. 
For the parabolic equation, the following result on existence and regularity holds true.

TheOREm 7.2. Suppose that (A5) is satisfied. Then, for every $u \in L^{2}(Q)$, the state equation (7.1) has a unique solution $y_{u} \in C(\bar{Q}) \cap W(0, T)$. If $u_{k} \rightarrow u$ weakly in $L^{2}(Q)$, then $y_{u_{k}} \rightarrow y_{u}$ strongly in $C(\bar{Q})$.

The proof of the theorem is postponed to section 7.4.

THEOREM 7.3. Assume that (A5)-(A8) are fulfilled, the function $L$ is convex with respect to the third component, and the set of feasible controls is nonempty. Then the control problem (PP) has at least one solution.

This theorem is a standard consequence of Theorem 7.2.

7.2. Necessary optimality conditions. Also here, the control-to-state mapping $G(u)=y_{u}, G: L^{2}(Q) \rightarrow C(\bar{Q}) \cap W(0, T)$, and the reduced objective functional $J$ are of class $C^{2}$ from $L^{\infty}(Q)$ to their image spaces, provided that assumptions (A5)(A8) are satisfied. Since this is known (see [5]) we state the associated derivatives for convenience below.

We define, for $v \in L^{2}(Q)$, the function $z_{v}$ as the unique solution to

$$
\left\{\begin{aligned}
\frac{d z_{v}}{d t}+A z_{v}+\frac{\partial f}{\partial y}\left(x, t, y_{u}\right) z_{v} & =v & & \text { in } Q \\
-\partial_{x} z_{v}(a, t) & =0 & & \text { in }(0, T) \\
\partial_{x} z_{v}(b, t) & =0 & & \text { in }(0, T) \\
y(x, 0) & =0 & & \text { in }(a, b)
\end{aligned}\right.
$$

Then $G^{\prime}(u), G: L^{2}(Q) \rightarrow C(\bar{Q}) \cap W(0, T)$, is given by $G^{\prime}(u) v=z_{v}$. Moreover, for $v_{1}, v_{2} \in L^{2}(Q)$, we introduce $z_{v_{i}}=G^{\prime}(u) v_{i}, i=1,2$, and obtain $G^{\prime \prime}(u) v_{1} v_{2}=z_{v_{1} v_{2}}$, where $z_{v_{1} v_{2}}$ is the solution to

$$
\left\{\begin{aligned}
\frac{d z_{v_{1} v_{2}}}{d t}+A z_{v_{1} v_{2}}+\frac{\partial f}{\partial y}\left(x, t, y_{u}\right) z_{v_{1} v_{2}}+\frac{\partial^{2} f}{\partial y^{2}}\left(x, t, y_{u}\right) z_{v_{1}} z_{v_{2}}=0 & \text { in } Q \\
-\partial_{x} z_{v_{1} v_{2}}(a, t)=0 & \text { in }(0, T) \\
\partial_{x} z_{v_{1} v_{2}}(b, t)=0 & \text { in }(0, T) \\
z_{v_{1} v_{2}}(x, 0)=0 & \text { in }(a, b)
\end{aligned}\right.
$$

The adjoint state $\varphi_{0 u} \in W(0, T)$ associated with $u$ and $J$ is introduced as the unique solution to

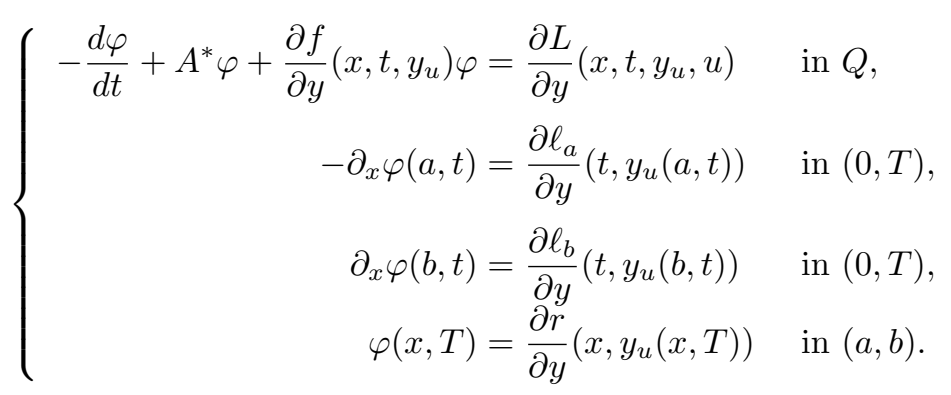

Copyright $@$ ㅇ by SIAM. Unauthorized reproduction of this article is prohibited. 
We have

$$
\begin{aligned}
J^{\prime}(u) v=\int_{Q}\left(\frac{\partial L}{\partial u}\left(x, t, y_{u}, u\right)+\varphi_{0 u}\right) v d x d t \\
J^{\prime \prime}(u) v_{1} v_{2}=\int_{Q}\left[\frac{\partial^{2} L}{\partial y^{2}}\left(x, t, y_{u}, u\right) z_{v_{1}} z_{v_{2}}+\frac{\partial^{2} L}{\partial y \partial u}\left(x, t, y_{u}, u\right)\left(z_{v_{1}} v_{2}+z_{v_{2}} v_{1}\right)\right. \\
\left.\quad+\frac{\partial^{2} L}{\partial u^{2}}\left(x, t, y_{u}, u\right) v_{1} v_{2}-\varphi_{0 u} \frac{\partial^{2} f}{\partial y^{2}}\left(x, t, y_{u}\right) z_{v_{1}} z_{v_{2}}\right] d x d t \\
+\int_{0}^{T} \frac{\partial^{2} \ell_{a}}{\partial y^{2}}\left(t, y_{u}(a, t)\right) z_{v_{1}}(a, t) z_{v_{2}}(a, t) d t \\
+\int_{0}^{T} \frac{\partial^{2} \ell_{b}}{\partial y^{2}}\left(t, y_{u}(b, t)\right) z_{v_{1}}(b, t) z_{v_{2}}(b, t) d t \\
+\int_{\Omega} \frac{\partial^{2} r}{\partial y^{2}}\left(x, y_{u}(x, T)\right) z_{v_{1}}(x, T) z_{v_{2}}(x, T) d x
\end{aligned}
$$

We require the following linearized Slater condition: There exists $u_{0} \in L^{\infty}(Q)$ with $\alpha(x, t) \leq u_{0}(x, t) \leq \beta(x, t)$ for a.e. $(x, t) \in Q$ such that

$$
g(x, t, \bar{y}(x, t))+\frac{\partial g}{\partial y}(x, t, \bar{y}(x, t)) z_{u_{0}-\bar{u}}(x, t)<0 \quad \forall(x, t) \in K .
$$

Notice that we have assumed (7.3), since this is needed to satisfy (7.9). The Hamiltonian $H$ is defined by

$$
H(x, t, y, u, \varphi)=L(x, t, y, u)+\varphi[u-f(x, t, y)],
$$

and the first-order necessary conditions admit the following form (see Casas [5]).

THEOREM 7.4. Let $\bar{u}$ be a local solution of (PP). Suppose that assumptions (A5)(A8) hold, and assume the Slater condition (7.9) with some $u_{0} \in L^{\infty}(Q), \alpha(x, t) \leq$ $u_{0}(x, t) \leq \beta(x, t)$ for a.e. $(x, t) \in Q$. Then there exist a measure $\bar{\mu} \in M(K)$ and a function $\bar{\varphi} \in L^{\tau}\left(0, T ; W^{1, \sigma}(\Omega)\right)$, for all $\tau, \sigma \in[1,2)$, with $\frac{1}{\tau}+\frac{1}{\sigma}>\frac{3}{2}$, such that

$$
\left\{\begin{aligned}
-\frac{d \bar{\varphi}}{d t}+A^{*} \varphi+\frac{\partial f}{\partial y}(x, t, \bar{y}) \bar{\varphi} & =\frac{\partial L}{\partial y}(x, t, \bar{y}, \bar{u})+\frac{\partial g}{\partial y}(x, t, \bar{y}) \bar{\mu}_{\left.\right|_{Q}}, \\
-\partial_{x} \bar{\varphi}(a, t) & =\frac{\partial \ell_{a}}{\partial y}\left(t, y_{u}(a, t)\right)+\frac{\partial g}{\partial y}(a, t, \bar{y}(a, t)) \bar{\mu}_{\left.\left.\right|_{\{a\} \times(0, T)}\right)}, \\
\partial_{x} \bar{\varphi}(b, t) & =\frac{\partial \ell_{b}}{\partial y}(t, \bar{y}(b, t))+\frac{\partial g}{\partial y}(b, t, \bar{y}(b, t)) \bar{\mu}_{\{b\} \times(0, T)}, \\
\bar{\varphi}(x, T) & =\frac{\partial r}{\partial y}(x, \bar{y}(x, T))+\frac{\partial g}{\partial y}(x, T, \bar{y}(x, T)) \bar{\mu}_{\left.\right|_{\Omega \times\{T\}}}
\end{aligned}\right.
$$

for a.a. $x \in(a, b), t \in(0, T)$, where $\bar{\mu}_{\left.\right|_{Q}}, \bar{\mu}_{\left.\right|_{\{a\} \times(0, T)},} \bar{\mu}_{\left.\right|_{\{b\} \times(0, T)}}$, and $\bar{\mu}_{\left.\right|_{\Omega \times\{T\}}}$ denote the restrictions of $\mu$ to $Q,\{a\} \times(0, T),\{b\} \times(0, T)$, and $\Omega \times\{T\}$, respectively,

$$
\int_{K}(z(x, t)-g(x, t, \bar{y}(x, t))) d \bar{\mu}(x, t) \leq 0 \forall z \in C(K) \text {, with } z(x, t) \leq 0 \forall(x, t) \in K,
$$

and, for almost all $(x, t) \in Q$,

$$
H(x, t, \bar{y}(x, t), \bar{u}(x, t), \bar{\varphi}(x, t))=\min _{s \in\left[\alpha_{\varepsilon_{\bar{u}}}(x, t), \beta_{\bar{\varepsilon}}(x, t)\right]} H(x, t, \bar{y}(x, t), s, \bar{\varphi}(x, t)),
$$

where $\alpha_{\varepsilon_{\bar{u}}}$ and $\beta_{\varepsilon_{\bar{u}}}$ are defined along the lines of Theorem 3.1.

Copyright $@$ ( ) by SIAM. Unauthorized reproduction of this article is prohibited. 
The Lagrange function is defined in a standard way by

$$
\begin{aligned}
\mathcal{L}(u, \mu)= & \int_{Q} L\left(x, t, y_{u}(x, t), u(x, t)\right) d x d t+\int_{0}^{T} \ell_{a}\left(t, y_{u}(a, t)\right) d t \\
& +\int_{0}^{T} \ell_{b}\left(t, y_{u}(b, t)\right) d t+\int_{K} g\left(x, t, y_{u}(x, t)\right) d \mu(x, t) .
\end{aligned}
$$

For convenience, we establish only the second-order derivative of $\mathcal{L}$ :

$(7.13)$

$$
\begin{aligned}
& \frac{\partial^{2} \mathcal{L}}{\partial u^{2}}(u, \mu) v_{1} v_{2} \\
& =\int_{Q}\left[\frac{\partial^{2} L}{\partial y^{2}}\left(x, t, y_{u}, u\right) z_{v_{1}} z_{v_{2}}+\frac{\partial^{2} L}{\partial y \partial u}\left(x, t, y_{u}, u\right)\left(z_{v_{1}} v_{2}+z_{v_{2}} v_{1}\right)+\frac{\partial^{2} L}{\partial u^{2}}\left(x, t, y_{u}, u\right) v_{1} v_{2}\right. \\
& \left.\quad-\varphi_{u} \frac{\partial^{2} f}{\partial y^{2}}\left(x, t, y_{u}\right) z_{v_{1}} z_{v_{2}}\right] d x d t+\int_{\Omega} \frac{\partial^{2} r}{\partial y^{2}}\left(x, y_{u}(x, T)\right) z_{v_{1}}(x, T) z_{v_{2}}(x, T) d x \\
& \quad+\int_{0}^{T}\left[\frac{\partial^{2} \ell_{a}}{\partial y^{2}}\left(t, y_{u}(a, t)\right) z_{v_{1}}(a, t) z_{v_{2}}(a, t)+\frac{\partial^{2} \ell_{b}}{\partial y^{2}}\left(t, y_{u}(b, t)\right) z_{v_{1}}(b, t) z_{v_{2}}(b, t)\right] d t \\
& \quad+\int_{K} \frac{\partial^{2} g}{\partial y^{2}}\left(x, t, y_{u}(x, t)\right) z_{v_{1}}(x) z_{v_{2}}(x) d \bar{\mu}(x, t),
\end{aligned}
$$

where $\varphi_{u}$ is the solution of (7.10), where $u$ is taken for $\bar{u}, y_{u}$ instead of $\bar{y}$, and $\mu$ for $\bar{\mu}$.

7.3. Second-order sufficient optimality conditions. With the prerequisites of the preceding section at hand, the extension of the second-order sufficient optimality conditions to the parabolic case is straightforward. We define the cone of critical directions associated with $\bar{u}$ by

$$
\begin{aligned}
& C_{\bar{u}}=\left\{h \in L^{2}(Q): h \text { satisfies (7.14), (7.15), and (7.16) below }\right\}, \\
& h(x, t)=\left\{\begin{array}{l}
\geq 0 \text { if } \bar{u}(x, t)=\alpha(x, t), \\
\leq 0 \text { if } \bar{u}(x, t)=\beta(x, t), \\
=0 \text { if } \bar{H}_{u}(x, t) \neq 0,
\end{array}\right. \\
& \frac{\partial g}{\partial y}(x, t, \bar{y}(x, t)) z_{h}(x, t) \leq 0 \text { if } g(x, t, \bar{y}(x, t))=0, \\
& \int_{K} \frac{\partial g}{\partial y}(x, t, \bar{y}(x, t)) z_{h}(x, t) d \bar{\mu}(x, t)=0 .
\end{aligned}
$$

The sufficient second-order optimality conditions for $\bar{u}$ are stated in the following result.

THEOREM 7.5. Let $\bar{u}$ be a feasible control of problem $(\mathrm{PP})$ that satisfies, together with the associated state $\bar{y}$ and $(\bar{\varphi}, \bar{\mu}) \in L^{\tau}\left(0, T ; W^{1, \sigma}(\Omega)\right) \times M(K)$ for all $\tau, \sigma \in[1,2)$, with $\frac{1}{\tau}+\frac{1}{\sigma}>\frac{3}{2}$, the first-order conditions (7.10)-(7.12). Assume in addition that there exist two constants $\omega>0$ and $\tau>0$ such that

$$
\begin{aligned}
& \frac{\partial^{2} L}{\partial u^{2}}(x, t, \bar{y}(x, t), \bar{u}(x, t)) \geq \omega \text { if }\left|\bar{H}_{u}(x, t)\right| \leq \tau \text { for a.e. }(x, t) \in Q, \\
& \frac{\partial^{2} \mathcal{L}}{\partial u^{2}}(\bar{u}, \bar{\mu}) h^{2}>0 \quad \forall h \in C_{\bar{u}} \backslash\{0\} .
\end{aligned}
$$

Copyright $@$ by SIAM. Unauthorized reproduction of this article is prohibited. 
Then there exist $\varepsilon>0$ and $\delta>0$ such that, for every admissible control $u$ of problem $(\mathrm{PP})$, the following inequality holds:

$$
J(\bar{u})+\frac{\delta}{2}\|u-\bar{u}\|_{L^{2}(Q)}^{2} \leq J(u) \quad \text { if } \quad\|u-\bar{u}\|_{L^{\infty}(Q)}<\varepsilon .
$$

The proof is analogous to the one of Theorem 4.1. We have to perform obvious modifications that are along the line of the ones explained in the proof of Theorem 6.5. Therefore, we skip these details.

7.4. Proof of Theorem 7.2. To prepare the proof of Theorem 7.2, we first state some results on maximal parabolic regularity of the elliptic differential operator $A$. In the one-dimensional case we study here, $A$ admits the form

$$
A=\frac{\partial}{\partial x}\left[a_{11}(\cdot) \frac{\partial}{\partial x}\right] \text {. }
$$

Let us consider $A$ on its natural domain

$$
D:=D(A)=\left\{w \in H^{2}(\Omega): \frac{\partial w}{\partial x}(a)=\frac{\partial w}{\partial x}(b)=0\right\}
$$

that is dense in $L^{2}(\Omega)$. It is known that, for all $\tau \in(0,1)$,

$$
D\left(A^{\tau}\right)= \begin{cases}H^{2 \tau}(\Omega) \cap\left\{w: \frac{\partial w}{\partial x}(a)=\frac{\partial w}{\partial x}(b)=0\right\} & \text { if } \tau>\frac{3}{4}, \\ H^{2 \tau}(\Omega), & \text { if } \tau<\frac{3}{4}\end{cases}
$$

cf. [24]. In particular, we have $D\left(A^{\frac{1}{2}}\right)=H^{1}(\Omega)$. To shorten the notation, we write below $S:=(0, T)$ with closure $\bar{S}$. Moreover, for a Banach space $X \subset L^{1}(\Omega)$ and $1<p<\infty$, we introduce the space

$$
W^{1, p}(S, X)=\left\{y \in L^{p}(S, X): \frac{\partial y}{\partial t} \in L^{p}(S, X)\right\} .
$$

It is known that, for all $1<p<\infty, A$ exhibits maximal parabolic $L^{p}\left(S, L^{p}(\Omega)\right)$ regularity. This means that, for all $f \in L^{p}\left(S, L^{p}(\Omega)\right)$, there is a unique solution $y \in W^{1, p}\left(S, L^{p}(\Omega)\right) \cap L^{p}(S, D(A))$ of

$$
\frac{\partial y}{\partial t}+A y=f \text { in } S, \quad y(0)=0,
$$

where the differential equation is to be understood in the distributional sense; cf. [13]. Here the definition of $D(A)$ must be adapted by replacing $W^{2, p}(\Omega)$ for $H^{2}(\Omega)$ in $(7.20)$. In all that follows, we apply this result with $p=2$ for $X=H:=L^{2}(\Omega)$. Therefore, for all $f \in L^{2}(S, H) \cong L^{2}(Q)$, there is a unique solution $y \in W^{1,2}(S, H) \cap L^{2}(S, D)$ of (7.21). The mapping $f \mapsto y$ is surjective and hence continuous.

Our proof relies on the following result.

Lemma 7.6. For all $0<\tau<\eta<1$ and $\kappa=\frac{\eta-\tau}{2 \eta}$, there holds the continuous injection $W^{1,2}(S, H) \cap L^{2}(S, D) \hookrightarrow C^{\kappa}\left(S, H^{\tau}(\Omega)\right)$.

Proof. We show first that $W^{1,2}(S, H) \hookrightarrow C^{\frac{1}{2}}(S, H)$. To this aim, let $y \in$ $W^{1,2}(S, H)$ and $t, s \in \bar{S}$ be given. Then

$$
\begin{aligned}
\|y(t)-y(s)\|_{H} & =\left\|\int_{s}^{t} y^{\prime}(\rho) d \rho\right\|_{H} \leq \int_{s}^{t}\left\|y^{\prime}(\rho)\right\|_{H} d \rho \\
& \leq\left(\int_{s}^{t}\left\|y^{\prime}(\rho)\right\|_{H}^{2} d \rho\right)^{\frac{1}{2}}\left(\int_{s}^{t} d \rho\right)^{\frac{1}{2}} \leq\|y\|_{W^{1,2}(S, H)}|t-s|^{\frac{1}{2}}
\end{aligned}
$$

Copyright $@$ by SIAM. Unauthorized reproduction of this article is prohibited. 
verifies the injection claimed above. Next, we prove the statement of the lemma. We denote by $[\cdot, \cdot]_{\theta}$ the complex interpolation functor; see Triebel [24]. It follows from $[2$, Chap. III, Thm. 4.10.2] and [24, Chap. 1.8] that the continuous injection

$$
W^{1,2}(S, H) \cap L^{2}(S, D) \hookrightarrow C\left(\bar{S},[H, D]_{1 / 2}\right)=C\left(\bar{S}, H^{1}(\Omega)\right)
$$

takes place. The interpolation identity $[H, D]_{1 / 2}=H^{1}(\Omega)$ is well known and can be found, for instance, in [24].

We fix now $\tau$ and $\eta$ by $0<\tau<\eta<1 / 2$ and put $\lambda=\tau / \eta$. Then we obtain with a generic constant $c$ that

$$
\begin{aligned}
\frac{\|y(t)-y(s)\|_{[H, D]_{\tau}}}{|t-s|^{\frac{1}{2}(1-\lambda)}} & \leq c \frac{\|y(t)-y(s)\|_{\left[H,[H, D]_{\eta}\right]_{\lambda}}}{|t-s|^{\frac{1}{2}(1-\lambda)}} \\
& \leq c \frac{\|y(t)-y(s)\|_{H}^{1-\lambda}}{|t-s|^{\frac{1}{2}(1-\lambda)}}\|y(t)-y(s)\|_{[H, D]_{\eta}}^{\lambda} \\
& \leq c\left(\frac{\|y(t)-y(s)\|_{H}}{|t-s|^{\frac{1}{2}}}\right)^{1-\lambda}\|y(t)-y(s)\|_{[H, D]_{\eta}}^{\lambda},
\end{aligned}
$$

where we have applied the complex reiteration theorem, [24, Chap. 1.9.3]. In the last estimate, the first factor is bounded, since $W^{1,2}(S, H) \hookrightarrow C^{\frac{1}{2}}(S, H)$. In view of the injection $(7.22)$ and $[H, D]_{\eta}=H^{2 \eta}(\Omega)$, with $0<2 \eta<1$, the second factor can be estimated by

$$
\begin{aligned}
\|y(t)-y(s)\|_{[H, D]_{\eta}}^{\lambda} & \leq c\|y(t)-y(s)\|_{H^{2 \eta}(\Omega)}^{\lambda} \leq\left(2 c\|y\|_{C\left(\bar{S}, H^{2 \eta}(\Omega)\right)}\right)^{\lambda} \\
& \leq\left(c\|y\|_{C\left(\bar{S}, H^{1}(\Omega)\right)}\right)^{\lambda} \leq c\|y\|_{W^{1,2}(S, H) \cap L^{2}(S, D)}^{\lambda} .
\end{aligned}
$$

In the last estimate, we have used the embedding (7.22). Moreover, we took advantage of the equivalence of the norms of $[H, D]_{\eta}$ and $H^{2 \eta}(\Omega)$. Therefore, the second factor in (7.24) is bounded, too. The statement of the lemma follows now from (7.24) after inserting $\tau:=2 \tau, \eta:=2 \eta,[H, D]_{\tau}=H^{2 \tau}(\Omega)$, and $\kappa=\frac{1}{2}(1-\lambda)$ into (7.23).

Proof of Theorem 7.2. The existence result of Theorem 7.2 is well known; we refer to Casas [5]. Therefore, we show only that weakly converging sequences of controls are transformed to strongly converging sequences of states.

Let a sequence $\left(u_{k}\right)$ be given that converges weakly in $L^{2}(Q)$ to $u$. Consider the equation for $y_{k}$ and $u_{k}$ :

$$
\begin{aligned}
\frac{\partial y_{k}}{\partial t}+A y_{k}+f\left(x, y_{k}\right) & =u_{k} & & \text { in } Q, \\
\frac{\partial y_{k}}{\partial x}(a, t) & =0 & & \text { in } S, \\
\frac{\partial y_{k}}{\partial x}(b, t) & =0 & & \text { in } S, \\
y_{k}(0) & =y_{0} & & \text { in } \Omega .
\end{aligned}
$$

Standard arguments show that $y_{k} \rightarrow y$ in $W(0, T) \cap C(\bar{Q})$, where $y=y_{u}$. The functions $y_{k}$ are uniformly bounded in $C(\bar{Q})$, hence the sequence $\left(d\left(\cdot, y_{k}\right)\right)$ is bounded in $L^{2}(Q)$, and we can select a weakly converging subsequence indexed by $k_{l}$. We write $f_{k}=u_{k}-d\left(\cdot, y_{k}\right)$ and split $y_{k}=v+w_{k}$, where $w_{k}$ solves

$$
\frac{\partial w_{k}}{\partial t}+A w_{k}=f_{k}
$$

Copyright (c) by SIAM. Unauthorized reproduction of this article is prohibited. 
with homogeneous inital and boundary conditions, while $v$ solves

$$
\frac{\partial v}{\partial t}+A v=0
$$

with inhomogeneous initial condition $v(0)=y_{0}$ and homogeneous boundary conditions. Thanks to Lemma 7.6, the sequence $\left(w_{k_{l}}\right)$ converges weakly in $C^{\kappa}\left(S, H^{\tau}(\Omega)\right)$, where $\kappa>0$ and $\tau>1 / 2$ can be chosen. Therefore, the functions $w_{k_{l}}$ belong to a space $C^{\sigma}(\bar{Q})$ with some positive $\sigma$ so that, by compact embedding into $C(\bar{Q})$, the sequence converges strongly in $C(\bar{Q})$. Consequently, $y_{k_{l}}=v+w_{k_{l}}$ converges strongly in $C(\bar{Q})$ towards $y$. Moreover, it follows by standard arguments that $y=y_{u}$. Since this holds for all subsequences with the same limit $y$, the whole sequence $\left(y_{k}\right)$ converges uniformly to $y_{u}$.

Acknowledgment. The authors are grateful to J. Rehberg (Weierstrass Institute Berlin (WIAS)) for pointing out the proof of Lemma 7.6.

\section{REFERENCES}

[1] J. J. Alibert and J. P. Raymond, Boundary control of semilinear elliptic equations with discontinuous leading coefficients and unbounded controls, Numer. Funct. Anal. Optim., 18 (1997), pp. 235-250.

[2] H. Amann, Linear and Quasilinear Parabolic Problems, Birkhäuser, Basel, 1995.

[3] F. Bonnans, Second-order analysis for control constrained optimal control problems of semilinear elliptic systems, Appl. Math. Optim., 38 (1998), pp. 303-325.

[4] F. Bonnans And A. Shapiro, Perturbation Analysis of Optimization Problems, SpringerVerlag, New York, 2000.

[5] E. Casas, Pontryagin's principle for state-constrained boundary control problems of semilinear parabolic equations, SIAM J. Control Optim., 35 (1997), pp. 1297-1327.

[6] E. CASAS, Necessary and sufficient optimality conditions for elliptic control problems with finitely many pointwise state constraints, ESAIM Control Optim. Calc. Var., to appear.

[7] E. Casas And M. Mateos, Second order optimality conditions for semilinear elliptic control problems with finitely many state constraints, SIAM J. Control Optim., 40 (2002), pp. 1431-1454.

[8] E. Casas, J. Raymond, And H. Zidani, Pontryagin's principle for local solutions of control problems with mixed control-state constraints, SIAM J. Control Optim., 39 (2000), pp. $1182-1203$.

[9] E. Casas and F. Tröltzsch, Second-order necessary optimality conditions for some stateconstrained control problems of semilinear elliptic equations, Appl. Math. Optim., 39 (1999), pp. 211-227.

[10] E. CASAS AND F. TRÖLTzSCh, Second-order necessary and sufficient optimality conditions for optimization problems and applications to control theory, SIAM J. Optim., 13 (2002), pp. 406-431.

[11] E. Casas, F. Tröltzsch, and A. Unger, Second order sufficient optimality conditions for a nonlinear elliptic control problem, J. Anal. Appl., 15 (1996), pp. 687-707.

[12] E. Casas, F. Tröltzsch, And A. Unger, Second order sufficient optimality conditions for some state-constrained control problems of semilinear elliptic equations, SIAM J. Control Optim., 38 (2000), pp. 1369-1391.

[13] G. DoRe, $l^{p}$-regularity for abstract differential equations, in Functional Analysis and Related Topics, in Proceedings of the International Conference in memory of Prof. K. Yosida held at RIMS, Kyoto University, 1991, Lecture Notes Math. 1540, H. Komatsu, ed., SpringerVerlag, Berlin, 1993, pp. 25-38.

[14] J. DunN, $l^{2}$ sufficient optimality conditions for end-constrained optimal control problems with inputs in a polyhedron, SIAM J. Control Optim., 36 (1998), pp. 1833-1851.

[15] D. Gilbarg and N. Trudinger, Elliptic Partial Differential Equations of Second Order, Springer-Verlag, Berlin, 1977.

[16] A. D. Ioffe, Necessary and sufficient conditions for a local minimum. 3: Second order conditions and augmented duality, SIAM J. Control Optim., 17 (1979), pp. 266-288.

[17] X. Li And J. Yong, Necessary conditions for optimal control of distributed parameter systems, SIAM J. Control Optim., 29 (1991), pp. 895-908. 
[18] K. MalAnOwski, Two-norm approach in stability and sensitivity analysis of optimization and optimal control problems, Adv. Math. Sci. Appl., 2 (1993), pp. 397-443.

[19] K. Malanowski, Sufficient optimality conditions for optimal control subject to state constraints, SIAM J. Control Optim., 35 (1997), pp. 205-227.

[20] H. Maurer, First and second order sufficient optimality conditions in mathematical programming and optimal control, Math. Program. Study, 14 (1981), pp. 163-177.

[21] H. Maurer and J. Zowe, First- and second-order conditions in infinite-dimensional programming problems, Math. Program., 16 (1979), pp. 98-110.

[22] J.-P. RAYmond And F. TrÖltzSch, Second order sufficient optimality conditions for nonlinear parabolic control problems with state constraints, Discrete Contin. Dyn. Syst., 6 (2000), pp. 431-450.

[23] G. Stampacchia, Problemi al contorno ellittici, con dati discontinui, dotati di soluzioni hölderiane, Ann. Mat., 51 (1960), pp. 1-37.

[24] H. Triebel, Interpolation Theory, Function Spaces, Differential Operators, North-Holland, Amsterdam, 1978.

Copyright (c) by SIAM. Unauthorized reproduction of this article is prohibited. 